\title{
Advances and potential new developments in imaging techniques for posterior uveitis. Part 1: noninvasive imaging methods
}

\author{
Ilknur Tugal-Tutkun $\mathbb{1}^{1} \cdot$ Carl P. Herbort $\mathrm{Jr}^{2} \cdot$ Alessandro Mantovani ${ }^{3} \cdot$ Piergiorgio Neri $\mathbb{\circledR}^{4,5} \cdot$ Moncef Khairallah $^{6}$
}

Received: 29 May 2020 / Revised: 18 June 2020 / Accepted: 19 June 2020 / Published online: 16 July 2020

(c) The Author(s), under exclusive licence to The Royal College of Ophthalmologists 2020

\begin{abstract}
The aim of this review was to identify the imaging methods at our disposal to optimally manage posterior uveitis at the present time. The focus was put on methods that have become available since the 1990s, some 30 years after fluorescein angiography had revolutionized imaging of posterior uveitis in particular imaging of the retinal vascular structures in the 1960s. We have focussed our review on precise imaging methods that have been standardized and validated and can be used universally thanks to commercially produced and available instruments for the diagnosis and follow-up of posterior uveitis. The first part of this imaging review will deal with noninvasive imaging methods, focusing on fundus autofluorescence and optical coherence tomography as well as recent developments in imaging of the posterior segment.
\end{abstract}

\section{Introduction}

While imaging techniques exploring posterior uveitis have made a spectacular progress in the last two to three decades, traditional mostly obsolete approaches are still put forward by official agencies, such as the Food and Drug Administration (FDA), failing to recommend precise and objective investigations, so hampering the diagnosis and management of posterior uveitis worldwide.

Ilknur Tugal-Tutkun

itutkun@yahoo.com

$\triangle$ Carl P. Herbort Jr

cph@herbortuveitis.ch

1 Department of Ophthalmology, Istanbul Faculty of Medicine, Istanbul University, Istanbul, Turkey

2 Retinal and Inflammatory Eye Diseases, Centre for Ophthalmic Specialized Care (COS), Clinic Montchoisi Teaching Centre, Lausanne, Switzerland

3 Ophthalmology Unit, Ospedale Valduce, Como, Italy

4 Cleveland Clinic Abu Dhabi, Abu Dhabi, UAE

5 Cleveland Clinic Lerner College of Medicine-Case Western Reserve University, Cleveland, OH, USA

6 Department of Ophthalmology, Fattouma Bourguiba University Hospital, Faculty of Medicine, University of Monastir, Monastir, Tunisia
The principal and sole outcome measure for studies on posterior uveitis recommended by the FDA is vitreous haze based on a system proposed more than 40 years ago [1] and taken over without change by the standardization of uveitis nomenclature (SUN) group [2]. It has since been shown that in most posterior uveitis entities, vitreous haze is minimal and unsuitable as an outcome measure. In inflammatory choriocapillaropathies, for example, such as multiple evanescent white dot syndrome (MEWDS), acute posterior multifocal placoid pigment epitheliopathy (APMPPE), idiopathic multifocal choroiditis (MFC), or serpiginous choroiditis, there is minimal or no vitreous inflammation; and vitreous haze grading cannot be used as a biomarker of activity. Precise follow-up will require noninvasive imaging, including fundus autofluorescence (FAF) and optical coherence tomography (OCT), and invasive imaging with indocyanine green angiography (ICGA) [3]. Another example is Vogt-Koyanagi-Harada (VKH) disease where vitreous haze is very rarely, if at all, seen in initial onset disease, and retinal and choroidal OCT as well as ICGA are the essential imaging methods for the diagnosis and monitoring of response to anti-inflammatory therapy [4]. The approach of these diseases was biased because the most adequate imaging methods for choroiditis were not taken into account [5]. A whole array of precise, quantitative, and objective imaging modalities are available today; and only widespread use of these modalities will improve standards of care in clinical practice as well as outcome measures in uveitis research [6-9]. 
The aim of this review is to concentrate on the progress made in the precision with which posterior uveitis can be investigated nowadays thanks to new or revisited techniques that have become available for routine use, such as laser flare photometry (LFP), fluorescein angiography (FA), ICGA and dual FA/ICGA scoring, FAF, and OCT, including enhanced depth imaging OCT (EDI-OCT), swept source OCT (SSOCT), and OCT angiography (OCT-A) [7-9].

In this part of the review on diagnostic imaging in posterior uveitis, we will explore the noninvasive methods, including LFP, FAF, and OCT, and we will also address recent developments like OCT-A imaging that still needs to be standardized and evaluated for routine use.

\section{Laser flare photometry (LFP)}

LFP measures backscattered photons from the aqueous proportional to the quantity of inflammatory (or other) proteins present in the same fashion as the slit lamp shows flare. The measurement units are photons per miliseconds ( $\mathrm{ph} / \mathrm{ms})$. Although it is not stricto sensu an imaging method and has no diagnostic value, it should be part of a comprehensive appraisal of posterior uveitis. Disruption of the blood-retina barriers in posterior uveitis entities leads to an overflow of proteins into the aqueous. Based on the extent and severity of posterior segment inflammation, varying levels of flare increase can be found in the absence of any clinical sign of anterior segment inflammation. In patients with clinically quiescent Behçet disease uveitis, flare levels correlate with retinal vascular/capillary leakage on FA $[10,11]$. In immunodeficient patients with CMV retinitis, a subclinical elevation of anterior chamber flare was found, which correlated with the area of involvement [12]. Thus LFP can be used as a measurement device of global inflammation (including posterior uveitis) with the exception of a few entities such as HLA-A29 birdshot retinochoroiditis (BRC) producing limited spill-over anterior chamber inflammation with values of $<15 \mathrm{ph} / \mathrm{ms}$ at presentation $[13,14]$.

\section{Fundus autofluorescence (FAF)}

\section{Blue autofluorescence (BAF)}

FAF, in particular BAF, has become one of the most interesting tools for the evaluation of the retina since the introduction of the superior imaging modality provided by the confocal scanning laser opthalmoscope (cSLO) that allows detection of low-intensity autofluorescence such as lipofuscin and melanin levels in the retinal pigment epithelium (RPE) cells.
In inflammatory disease, FAF images are obtained with blue light wavelength (BAF, excitation $488 \mathrm{~nm}$, emission $>500 \mathrm{~nm}$ ) [15]. BAF imaging is a technique that became available recently for assessing the RPE function and the integrity of the chorioretinal interface. It is generated from the bisretinoids of lipofuscin in the RPE cells. This material, the major fluorophores in the eye, is a mixture of several bisretinoids (A2E, A2PE) that are by-products of the visual cycle. These bisretinoids form primarily in the photoreceptor outer segments and are deposited secondarily in the RPE cell lysosomes during the process of photoreceptor outer segment phagocytosis.

The intensity of the autofluorescent signal is modified by the variation of the amount of the fluorophores and by the absorption of light by macular pigments and by RPE melanin [16]. Moreover BAF is also related to the photopigments in the photoreceptor outer segments. Under normal conditions, visual photopigments absorb the exciting blue light thereby attenuating the autofluorescent signal coming from RPE.

After blue light irradiation, during the photobleaching process, absorption of photons by photopigments induces the isomerization of 11-cis retinal to all-trans retinal conformation producing a reduction of the photopigment density with increased visualization of the autofluorescence signal coming from RPE. Thus, in case of damage to the photoreceptors, reduction of the photopigment density will lead to an increase of normal underlying RPE autofluorescence.

More recently, in inflammatory chorioretinal diseases, the interest of FAF analysis has emerged especially for inflammatory diseases of the choriocapillaris characterized by inflammatory hypo or nonperfusion of the choriocapillaris. This leads to ischaemic consequences at the level of the RPE and outer retina. Among these diseases, increased $\mathrm{BAF}$ is more pronounced on the more benign side of the spectrum such as in MEWDS and similar inflammatory choriocapillaropathies or in entities with smaller chorioretinal lesions such as MFC [3, 17]. A case of primary inflammatory choriocapillaropathy of the MFC type subjected to a complete ophthalmic evaluation using Spectralis HRA + OCT (Heidelberg Engineering, Heidelberg, Germany) is shown in Fig. 1.

In summary, FAF imaging is a fast, easy to perform, noninvasive technique providing an essential diagnostic tool for the investigation of inflammatory choriocapillaropathies (primary choriocapillaritis entities) [18]. In fact, in a few seconds, even in absence of the typical ophthalmoscopic and fluorescein angiographic findings, patterns of increased BAF help to suspect an inflammatory choriocapillaropathy; ICG angiographic findings will clear up all doubts. Autofluorescent images should be routinely acquired. Low-intensity BAF obtained by cSLO is an 


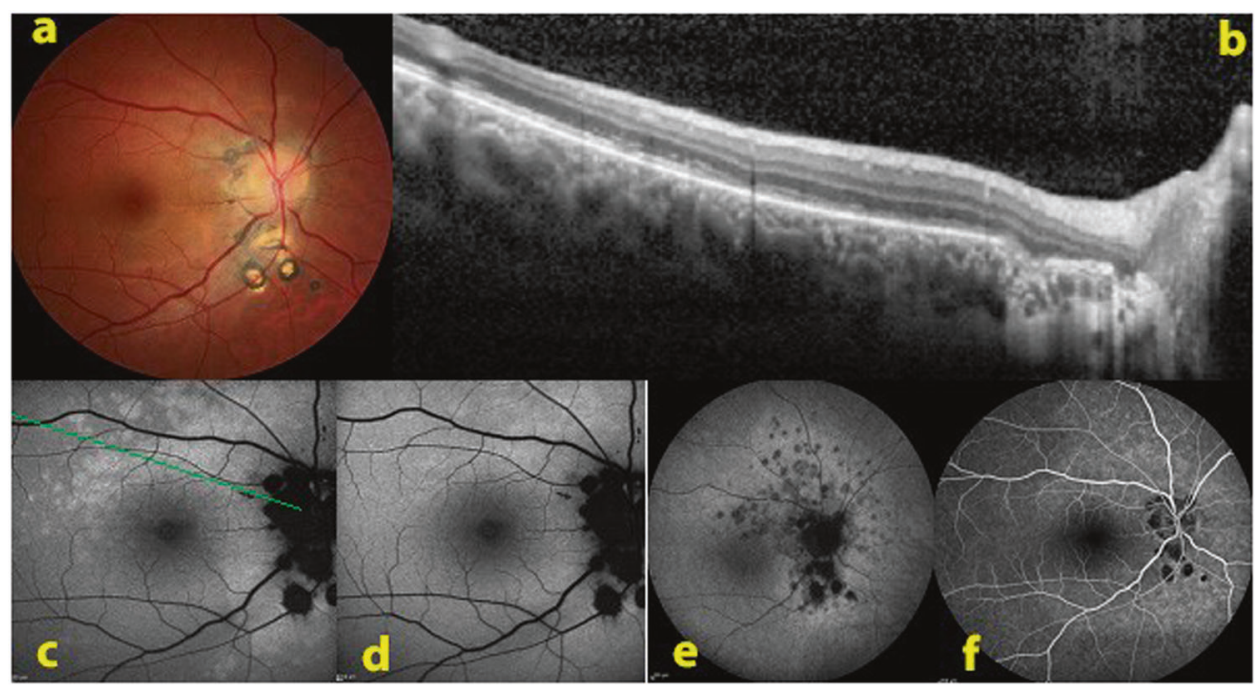

Fig. 1 Fundus autofluorescence in a case of multifocal choroiditis relapse. a Baseline colour ophthalmoscopic image showing chorioretinal scars around the optic disc. b SD-OCT with disruption of the ellipsoid zone through the bright autofluorescent area. c BAF imaging showing increased autofluorescence around the optic disc and above the macula. d This hyperautofluorescence disappeared after

imaging modality giving information on the chorioretinal interface integrity and function; the intensity of the autofluorescent signal depends on both the amount of lipofuscin in the RPE cells and the absorption of blue light by photoreceptor and macular pigments and by RPE melanin. Particularly in the choriocapillaritis entities of mild grade (MEWDS and MFC), active disease is often reflected by bright autofluorescent patterns; this is especially significant because sometimes funduscopy shows absence of pathology and FA may not be relevant. In this type of choriocapillaritis, increased BAF identifies photoreceptor damage rather than the accumulation of fluorophores as the cause of hyperautofluorescence.

Potential promising developments of FAF lie in the prospect of quantifying autofluorescence intensity. In the field of posterior uveitis, Boudreault et al. [19] recently used quantitative FAF (qFAF) to objectively measure shortwavelength autofluorescence intensity of fundus lesions in acute zonal occult outer retinopathy (AZOOR) indicating that this technique may be useful to understand the pathogenesis of the disease. Further developments, standardization, and routine accessibility of qAF might result in significant progress in noninvasive imaging of posterior uveitis [20].

\section{Optical coherence tomography (OCT)}

Since its introduction in 1991 [21], OCT technology has rapidly progressed from time-domain (TD-OCT) to SD- photobleaching process. e ICGA late frame showing confluent dark hypofluorescent area around the optic disc and numerous hypofluorescent dots representing areas of new inflammatory involvement. The optic disc and the chorioretinal scars were black. f FA showing only small hypofluorescent areas under the optic disc corresponding to chorioretinal scars.

OCT and SS-OCT, and these advanced noninvasive imaging modalities are now routinely used in uveitis practice [22]. OCT allows an 'optical biopsy' of the posterior segment structures with a high resolution, reproducibility, and precision that correlates well with histology sections. Mapping of individual retinal layers as well as volume rendering with 3-dimensional imaging have become possible.

In uveitis practice, OCT is used for the detection and monitoring of macular oedema, vitreomacular surface abnormalities, and structural damage to the macula. It is extremely helpful for the differential diagnosis of retinochoroidal inflammatory lesions and for monitoring response to therapy. Choroidal OCT imaging has allowed noninvasive visualization of choroidal granulomas and diffuse choroiditis as well as quantitative follow-up of choroidal thickness and structural changes in the choroid (see next chapter). OCT technology is also being investigated for the objective quantitative measurement of vitreous haze.

\section{OCT imaging of uveitic macular oedema}

Markomichelakis et al. [23] identified three distinct OCT patterns of fluid accumulation in patients with uveitis, including diffuse macular oedema (DMO), cystoid macular oedema (CMO), and concomitant serous retinal detachment (Fig. 2a, b), which were found to be similar to the patterns of diabetic macular oedema. It is of particular note that subfoveal fluid accumulation can only be detected by OCT, but not by FA imaging. 


\section{Fig. 2 SD-OCT showing} macular oedema in patients with uveitis. SD-OCT scans show diffuse macular oedema with serous retinal detachment in the left eye of a patient with Behçet uveitis (a); cystoid macular oedema with serous retinal detachment in the left eye of a patient with intermediate uveitis (b); and persistent serous retinal detachment as well as a few remaining inner nuclear layer cysts and epiretinal membrane in the left eye of a patient with sarcoid uveitis (c).
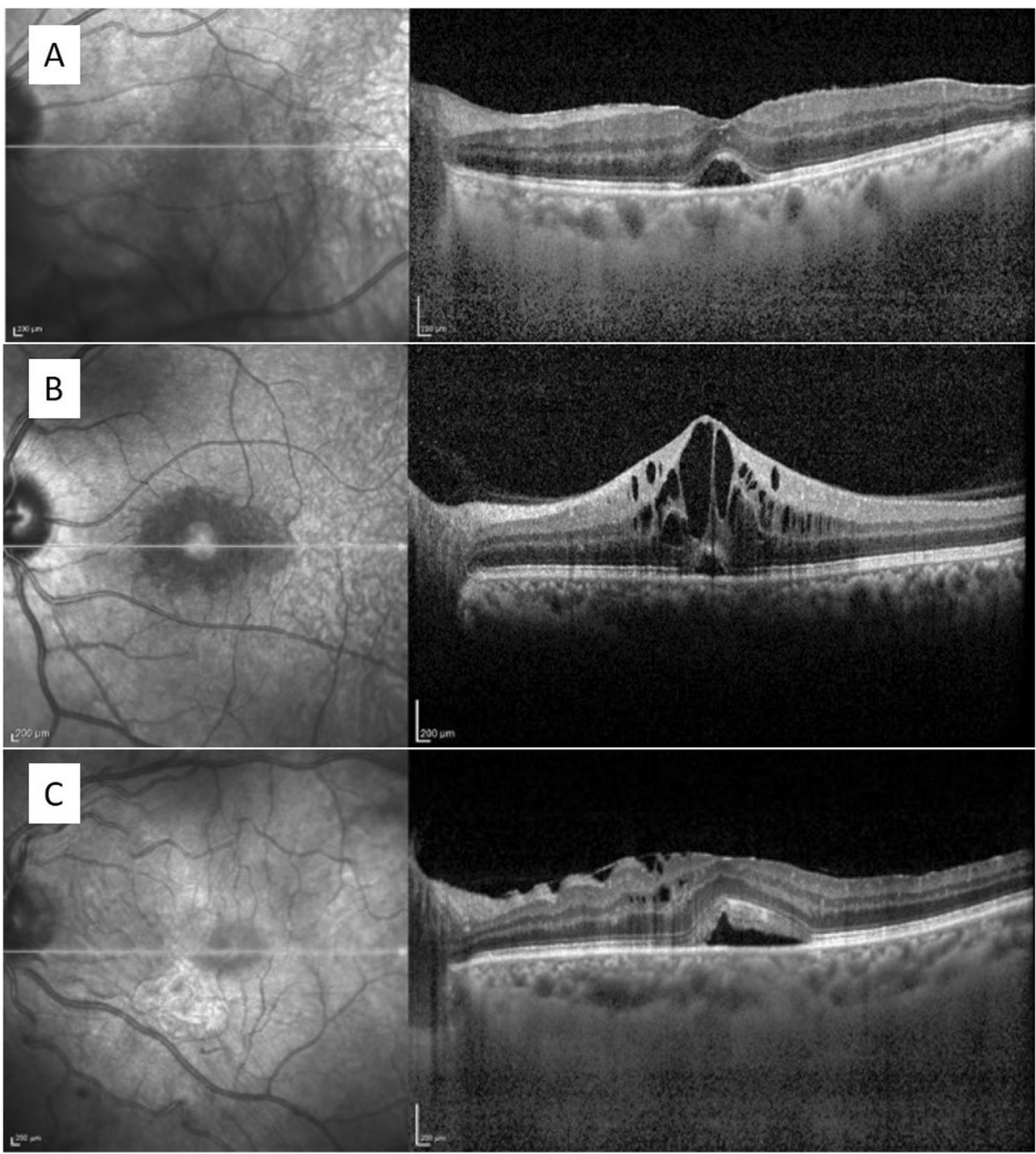

Another morphologic feature of macular oedema detected by OCT is the vitreoretinal interface abnormality in the form of epiretinal membrane (ERM) formation or vitreomacular traction (VMT) [24]. In a series of 500 consecutive uveitis patients who underwent standard SDOCT imaging of the macula, CMO was reported in $25 \%$, DMO in $11 \%$, serous retinal detachment in $8 \%$, and ERM with or without retinal surface wrinkling in $18 \%$ and $13 \%$, respectively [25]. Eyes with intermediate uveitis had highest central retinal thickness $(380 \pm 139 \mu \mathrm{m})$ and most frequent occurrence of CMO (40\%) [25]. In a recent review article that described the differentiating morphological features of macular oedema due to a variety of underlying pathologies, uveitic macular oedema was reported to be typically of cystoid morphology with central, concentric, and symmetric features, starting with small cysts in the inner nuclear layer and expanding to the outer nuclear layer, followed by accumulation of subretinal fluid; and the resolution of the latter lagged behind the resolution of outer and inner nuclear cysts [26] (Fig. 2c).

OCT permits not only the detection of these morphologic changes, but also quantitative analysis of macular thickness, which is especially useful in objective monitoring of response to treatment by the use of follow-up scan protocols. A $20 \%$ change in retinal thickness has been reported to be associated with clinically important changes in visual acuity [27]. Furthermore, using high-resolution OCT imaging, predictors of poor visual recovery such as atrophy and disorganization of inner retinal layers and disruption of outer retinal layers can be documented (Fig. 3) and may explain poor visual acuity despite resolution of intraretinal and subretinal fluid [28].

The presence of an ERM or VMT may be the cause of treatment failure as well and will be most reliably detected by OCT [29] (Fig. 4). OCT-based classification and definitions have been developed for the pathologies of vitreomacular interface, including vitreomacular adhesion, 
Fig. 3 Documentation of retinal damage by SD-OCT imaging in a case of chronic uveitic cystoid macular oedema. SD-OCT shows chronic cystoid macular oedema (a) and disruption of retinal layers after resolution of oedema (b) in the right eye of a patient with intermediate uveitis.
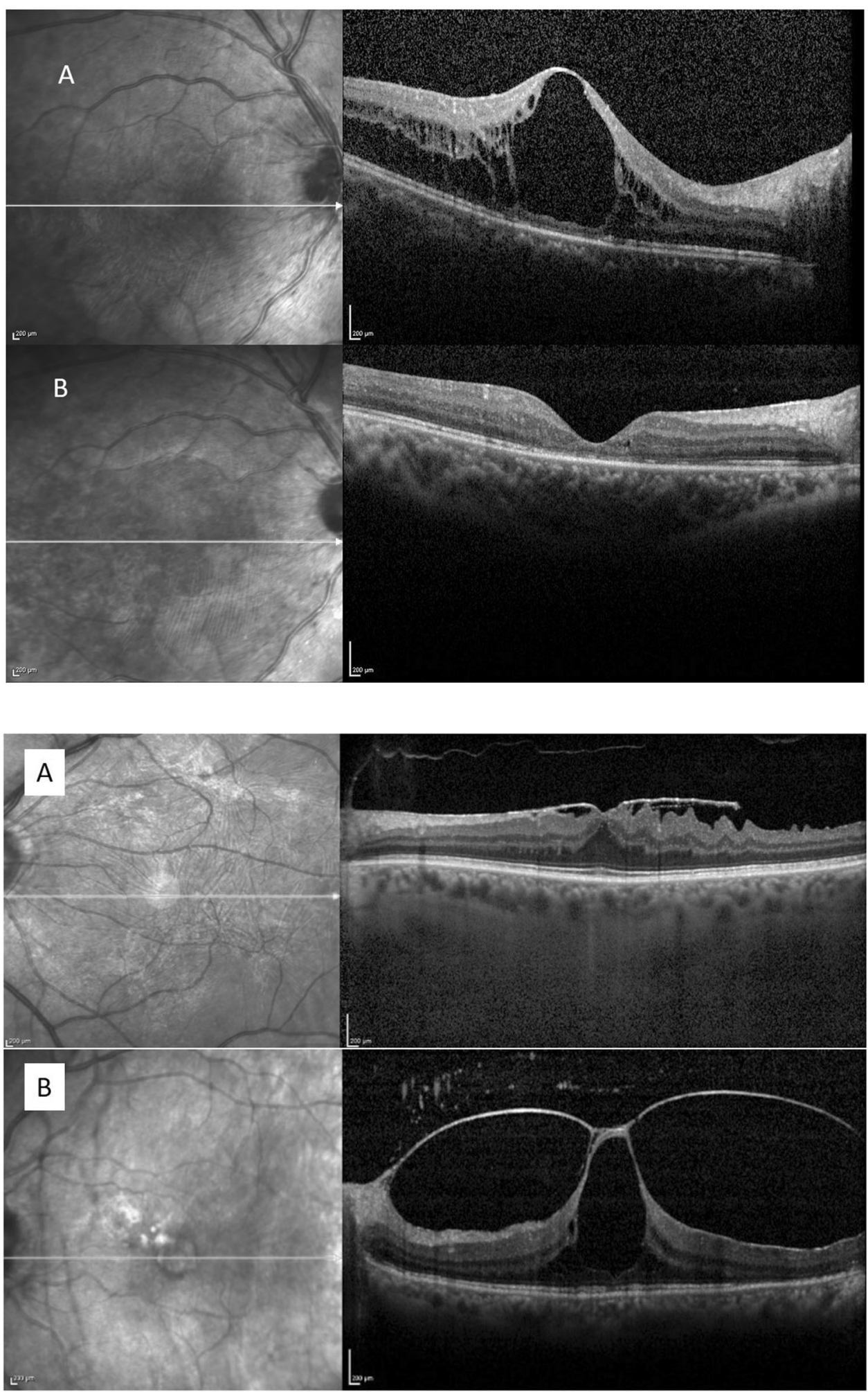

Fig. 4 SD-OCT showing vitreomacular interface abnormalities in patients with uveitis. SD-OCT scans show an epiretinal membrane causing elevation of the fovea and retinal wrinkling in the left eye of a patient with intermediate uveitis (a); vitreomacular traction in the left eye of a patient with sarcoid uveitis (b). traction, lamellar, and full-thickness macular holes as well as macular pseudohole [30]. In the Multicenter Uveitis Steroid Treatment (MUST) trial, ERM was found in $27.8 \%$ and VMT in $2.4 \%$ of 453 time-domain OCTs obtained at baseline [31]. In a series of 598 patients with uveitis seen at a single centre, $41 \%$ were found to have ERM in at least one eye on SD-OCT imaging, which was associated with lower visual acuity [32]. 


\section{OCT characterization of posterior uveitic entities}

OCT demonstrates characteristic patterns of a variety of posterior segment inflammatory lesions and thus constitutes an integral component of multimodal imaging of posterior uveitis. Both cross-sectional and en face OCT images help in delineation of inflammatory involvement of retino-choroidal layers. Evolution of inflammatory lesions and their sequelae and scars can be depicted by serial OCT imaging. The inner, outer, or full-thickness retinal, retino-choroidal, or choroidal involvement of inflammatory lesions at the posterior pole can be quickly and accurately identified by OCT imaging which greatly helps in the differential diagnosis of noninfectious and infectious posterior uveitis entities (Fig. 5).
Fig. 5 SD-OCT (EDI-OCT) characteristics of inflammatory lesions. a A white infiltrate at the posterior pole in the left eye of a patient with Behçet uveitis is characterized by a hyperreflective focal retinal thickening with shadowing beneath the lesion. b Follow-up OCT scan through the same spot after resolution of the retinal infiltrate shows a "pseudo-fovea" inferior to the fovea due to inner retinal atrophy and outer plexiform layer elevation as seen in the close-up image. Also note development of a slit-like retinal nerve fibre layer defect secondary to this lesion, indicated by white arrowheads both on the infrared image and the colour photograph. c Toxoplasma retinochoroiditis is characterized by full-thickness hyperreflectivity and disruption of the retinal layers in the necrotic area with shadowing and choroidal thickening beneath the lesion. d A choroidal granuloma is characterized by hyporeflective thickening of the choroid with secondary serous retinal detachment.

e Hyperautofluorescent active frond in serpiginous choroiditis is characterized by focal choroidal thickening and hyperreflective deposits in the outer retina (yellow arrows) and the hypoautofluorescent scarred region is characterized by loss of outer retinal layers and RPE as well as an increased transmission effect with hyperreflective thinned choroid (red arrows).
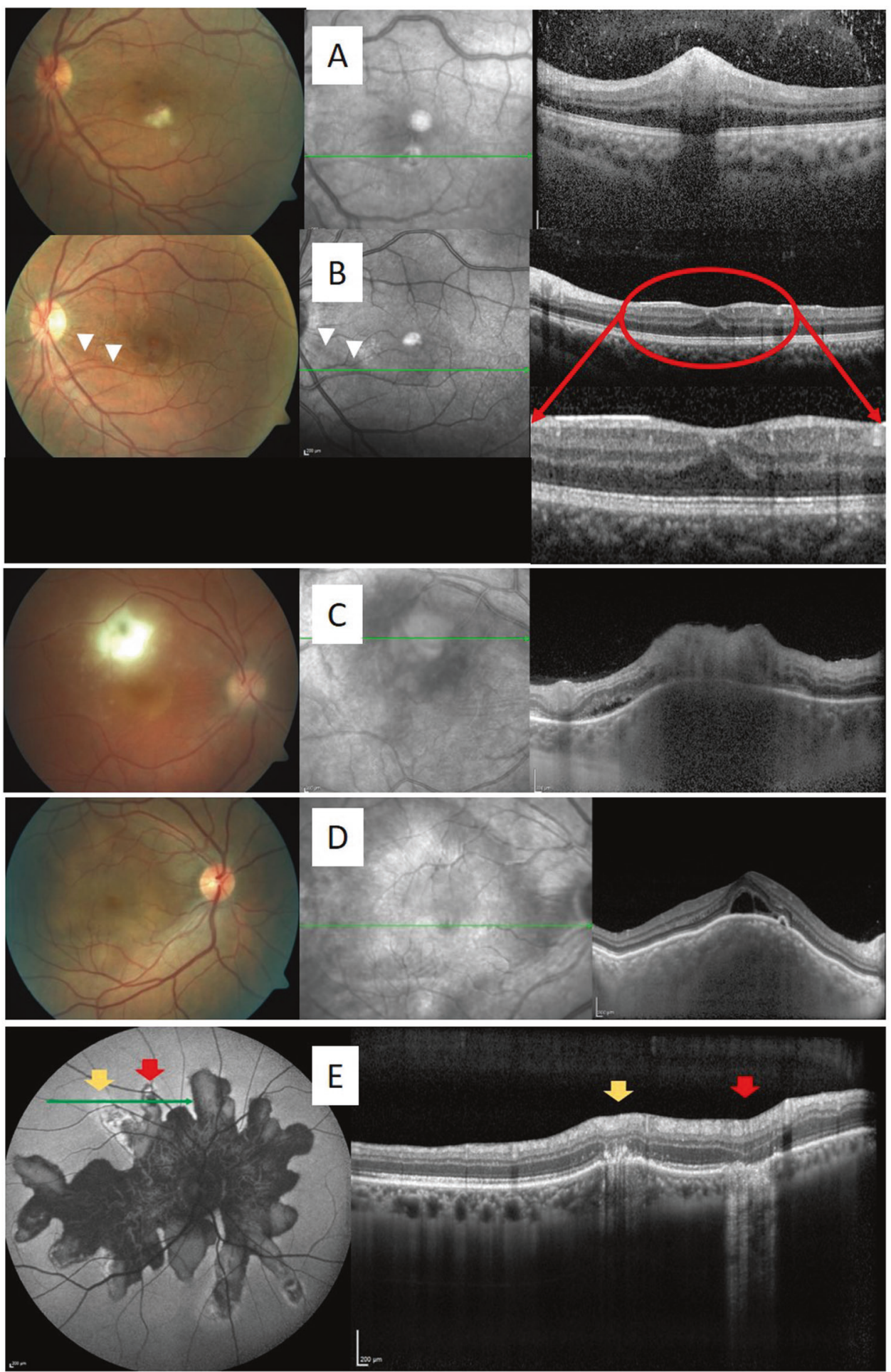

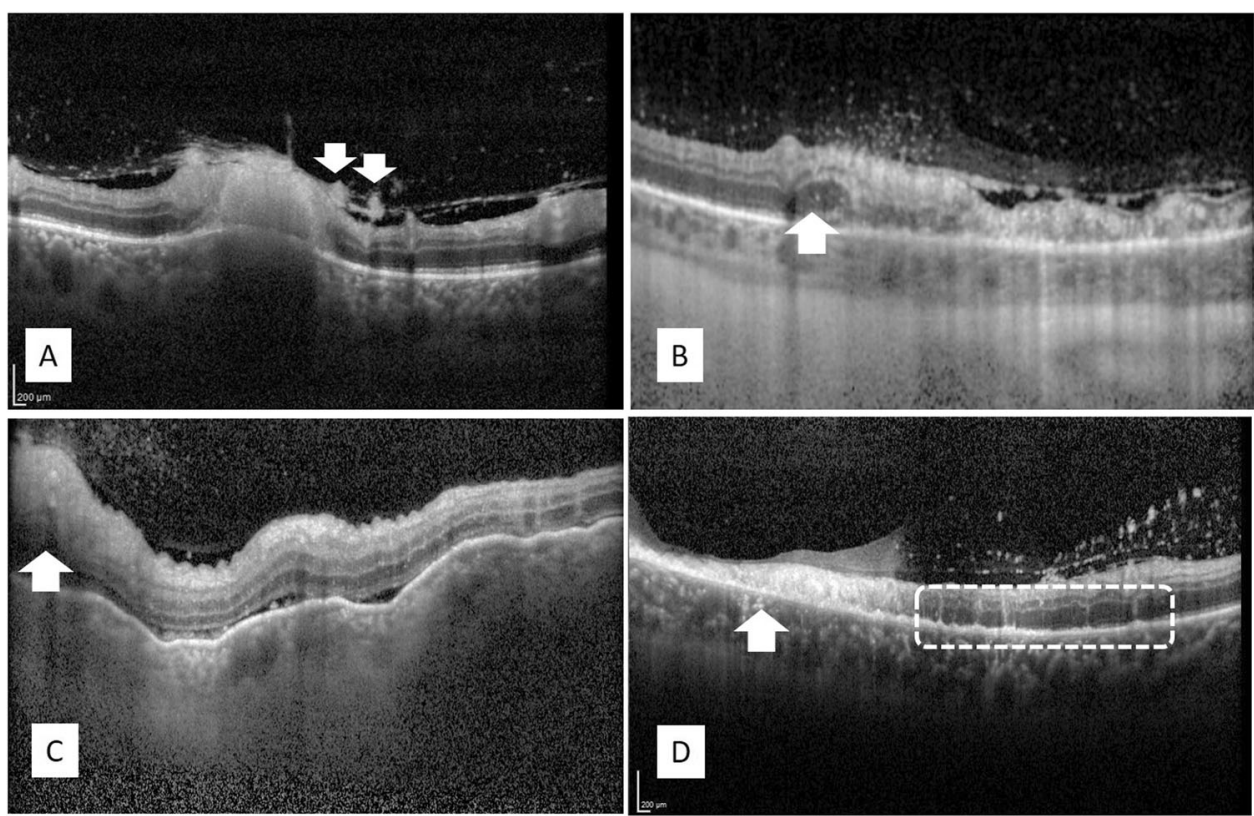

Fig. 6 OCT characteristics of infectious retinitis. a Toxoplasma retinochoroiditis: full-thickness hyperreflectivity of the retinal lesion with shadowing and thickening of the underlying choroid and hyperreflective round deposits along the posterior hyaloid (arrows); b syphilitic retinitis: full-thickness retinal hyperreflectivity and disruption of retinal layers, posterior hyaloidal deposits, and outer nuclear layer thickening at the advancing edge of the lesion (arrow); c VZV necrotizing retinitis: full-thickness retinal hyperreflectivity with

Inner retinal infiltrates that resemble cotton-wool spots are typically seen in Behçet disease uveitis and also in infectious entities such as bartonellosis, borreliosis, rickettsiosis as well as West Nile virus and chikungunya virus infections [33-37]. These infiltrates demonstrate the characteristic OCT features of hyperreflective focal retinal thickening with smudging of especially inner retinal layers and shadowing beneath the lesion without underlying choroidal thickening [33-37]. There may be an associated serous retinal detachment, especially with large lesions [33]. After the resolution of active lesions, follow-up OCT sections show inner retinal atrophy (indentation) and elevation of the outer plexiform layer [38, 39] (Fig. 5b). A retinal nerve fibre layer defect may also appear as a consequence of such lesions at the posterior pole [38]. While recurrences of inner retinal infiltrates are typically seen in active Behçet disease uveitis [34], infectious entities are uniphasic [33, 35-37].

Invernizzi et al. [40] have described the differentiating OCT features of infectious retinitis due to toxoplasmosis or herpes viruses. While all eyes had full-thickness hyperreflectivity and disruption of the retinal layers in the necrotic area, with a variable degree of vitritis and choroidal thickening, hyporeflectivity of the choroid beneath the necrotic retinal focus, hyperreflective oval deposits on the inner overlying vitreous cells (arrow), advancing with diffuse inner retinal hyperreflectivity, shallow subretinal fluid, and RPE undulations with thickened choroid; $\mathbf{d}$ CMV retinitis: Thinned retina with full-thickness retinal hyperreflectivity and disruption of retinal layers (arrow), posterior hyaloidal hyperreflective dots, and hyperreflective vertical strips within the outer nuclear layer between the necrotic and healthy retina (indicated by a rectangle), and hyperreflective dots in the choroid.

retinal surface as well as along the posterior hyaloid and retro-hyaloid hyperreflective spots were more commonly observed in toxoplasma retinochoroiditis. Intraretinal oedema and hyperreflective vertical strips within the outer nuclear layer were exclusively seen in viral retinitis [40]. Examples of infectious retinitis are shown in Fig. 6.

Involvement of the outer retinal layers is demonstrated by OCT in MEWDS, APMPPE, serpiginous choroiditis, MFC, and AZOOR [22]. While these changes are reversible in MEWDS [41, 42], permanent damage to the outer retina has been shown by OCT studies in the other entities [22]. Characteristic OCT features of active lesions in serpiginous choroiditis consist of uniform hyperreflectivity of the outer retina with disruption of outer retinal layers and RPE as well as hyperreflectivity and focal thickening of the underlying choroid. The inner retinal layers remain intact. The scarred area shows the loss of outer retinal layers and increased transmission effect [43]. Five-stage evolution of PIC that has been defined by SD-OCT imaging includes choroidal infiltration, formation of sub-RPE nodules, and then chorioretinal nodules, regression, and retinal herniation [44] (Fig. 7). SD-OCT also showed the retinal evolution in HLA-A29 BRC from the early oedematous stage to the late atrophic stage when disease is undertreated [45] (Fig. 8). 

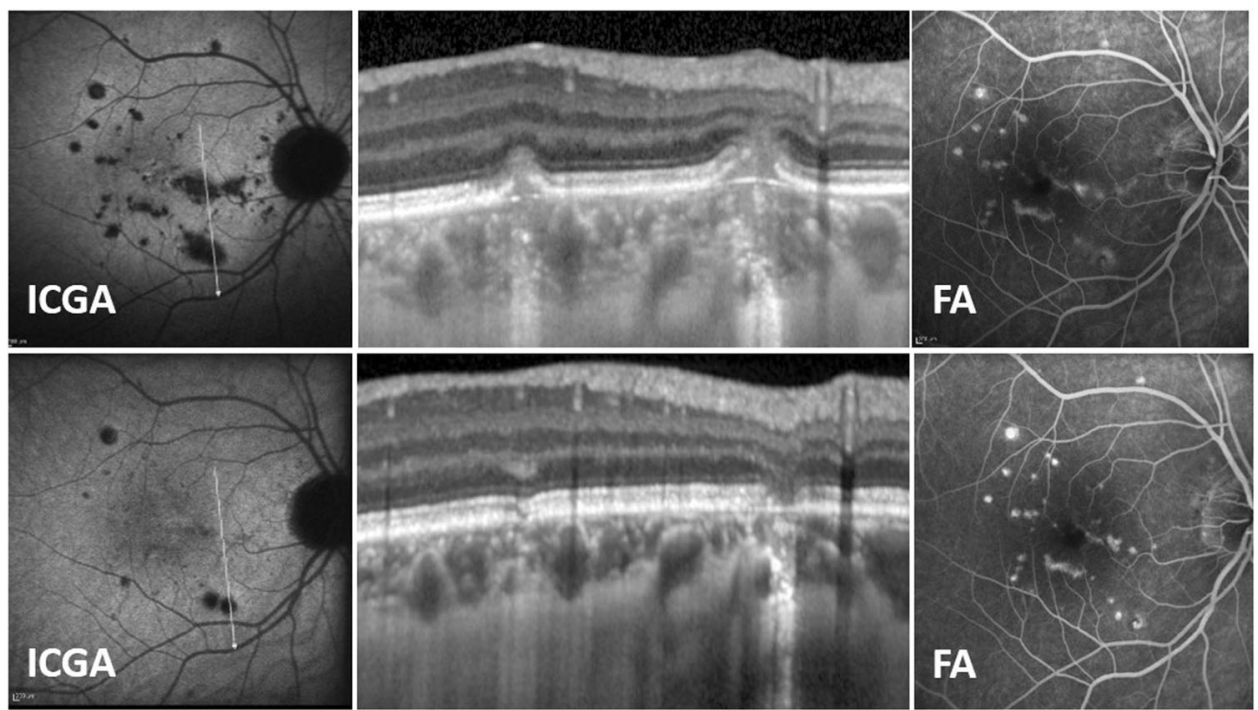

Fig. 7 ICGA/SD-OCT and FA images in a case of multifocal choroiditis. During the active phase of the disease (top row), OCT imaging discloses the presence of two inflammatory lesions showing elevation of the RPE by homogeneous material; RPE layer appears interrupted at its peak with material infiltrating the subretinal space without the involvement of the inner retina. Choroidal hyperreflectivity is well demonstrated here. In the quiescent phase (bottom row), highlighted by the disappearance of the ICGA hypoflurescent dark areas in the late frame, except those corresponding to the atrophic scars, OCT shows two scarred lesions with the resolution of the subRPE material. It is also interesting to note that the FA images do not permit to distinguish the active phase from the quiescent phase of the disease.
Fig. 8 SD-OCT showing the retinal evolution in HLA-A29 birdshot retinochoroiditis. OCT scans show evolution of retinal morphology from acuteearly oedematous stage (top) to intermediate stage with oedematous and atrophic areas (middle) and late atrophic stage (bottom). Evolution of mean retinal thickness is shown on right graph.

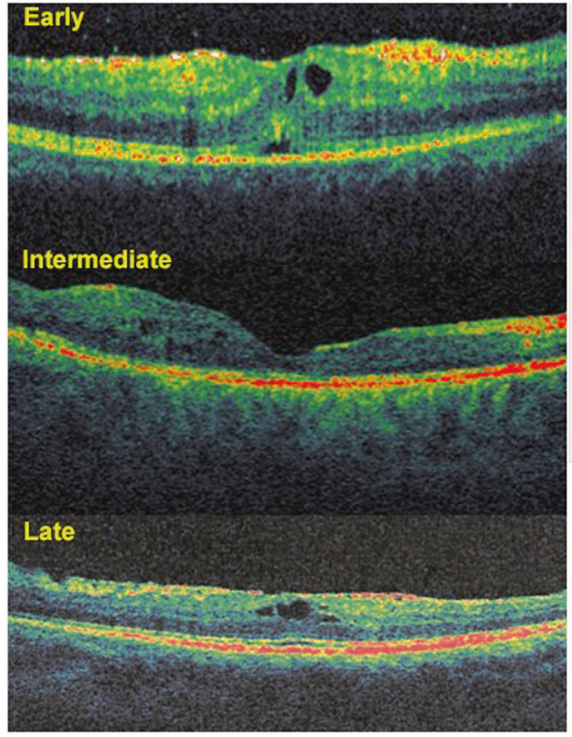

Serous retinal detachment due to diffuse choroidal inflammation is the hallmark of VKH and sympathetic ophthalmia. Multilobular serous retinal detachment with subretinal septae and undulations or inward bulging of RPE are the typical retinal OCT findings in acute VKH disease [46-48] (Fig. 9a). The resolution of serous retinal detachments with high-dose corticosteroid therapy can be closely monitored by noninvasive OCT imaging in these entities.

\section{Enhanced depth imaging OCT (EDI-OCT) for choroidal imaging}

In 2008, Spaide et al. described a method that allowed to obtain images of the choroid using conventional SD-OCT and enabled to measure thickness of the choroid [49]. They called the technique EDI-OCT and the method would prove especially useful for inflammatory diseases of the choroidal stroma such as VKH disease and HLA-A29 BRC as well as 
Fig. 9 Retinal and choroidal OCT features in acute VogtKoyanagi-Harada disease. SD-OCT shows serous retinal detachment with subretinal septae in the left eye of a patient with acute

Vogt-Koyanagi-Harada disease (a). EDI-OCT in another acute case of Vogt-Koyanagi-Harada disease shows substantial thickening of the choroid 511-537 $\mu \mathrm{m}$ (b).
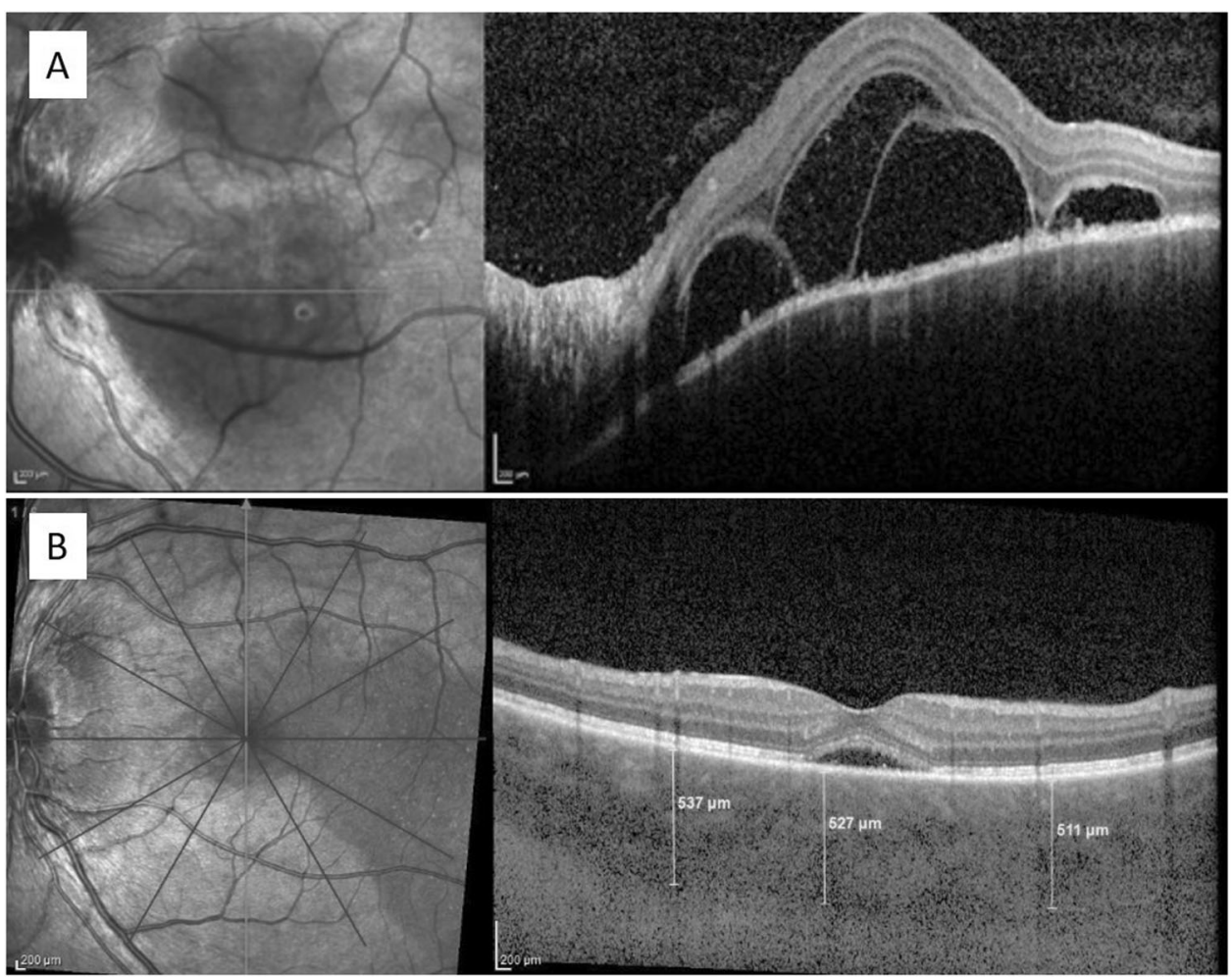

others [50]. Since its first description, the technique has been extensively used as it is readily available for routine investigation in currently commercialized OCT instruments such as the Heidelberg Spectralis OCT and other brands.

There is no doubt that EDI-OCT has taken a great importance in the exploration of choroiditis. Numerous reports on its use in primary stromal choroiditis (choroiditis entities starting electively in the choroidal stroma) including VKH, BRC, and sympathetic ophthalmia [51] and in other choroiditis entities such as sarcoidosis or tuberculous chorioretinitis have become available [52]. In acute VKH disease, reports are concordant and show that choroidal thickening is a characteristic EDI-OCT sign and a modality to monitor the effect of therapy and evolution of the choroidal disease [53, 54] (Fig. 9b). However, mainly because the technique, at its present stage, only gives information on the posterior pole, EDI-OCT is less sensitive for close follow-up of primary stromal choroiditis and to adjust treatment when compared to ICGA [55-57]. Nevertheless EDI-OCT has the advantage over ICGA to be a noninvasive method that can easily be repeated and that can be used to monitor choroidal inflammation when ICGA is not available. Like ICGA, EDI-OCT was able to detect occult choroidal inflammation in the absence of clinical signs or before clinical signs became apparent [58-60].

In chronic VKH, EDI-OCT findings are more difficult to interpret. Chronically evolving disease was shown to produce choroidal atrophy in the long term [61]. Another study showed that choroidal thickness was again increasing when disease activity was detected by ICGA [62]. So in chronic disease, atrophic areas of choroid can coexist with areas presenting recurrent inflammation and global choroidal thickness was not accounting clearly for the stage of evolution/inflammation. It was shown to be less reliable than ICGA and LFP measurement of intraocular inflammation to detect inflammatory activity [63].

In HLA-A29 BRC, where choroidal inflammation is developing in a similar fashion to VKH, EDI-OCT was shown to give useful information on the choroiditis [64, 65], but was also less sensitive than ICGA to monitor choroidal inflammatory activity [55-57]. Follow-up of choroidal thickness by EDI-OCT in BRC showed that it decreased with time, which could be prevented when aggressive and sustained treatment was applied [66] (Fig. 10). Unlike VKH and BRC, sarcoidosis can be qualified as a secondary stromal choroiditis, as choroiditis is a chance localization of a systemic disease and therefore lesions have a random location and global information on choroidal involvement is best obtained by ICGA, as it visualizes the whole fundus [67]. Sarcoid choroidal granulomas can be localized and monitored using EDI-OCT [68].

Even in inflammatory conditions that did not seem to involve the choroid such as Behçet uveitis, EDI-OCT revealed choroidal thickening $[69,70]$.

Although less sensitive than ICGA, EDI-OCT has become very popular for the appraisal of choroiditis as it is 

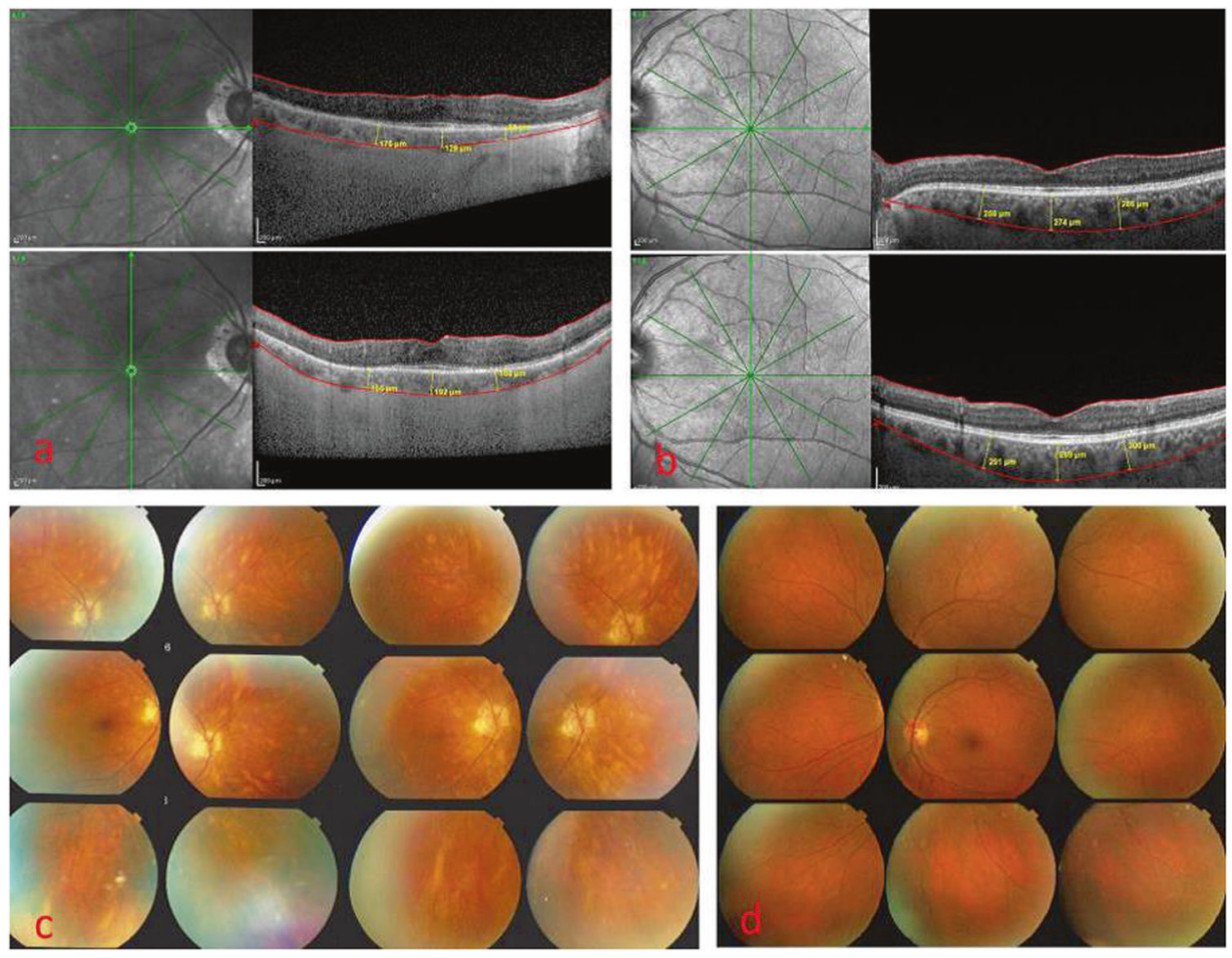

Fig. 10 EDI-OCT and fundus photograph in a chronic case of HLA-A29 birdshot retinochoroiditis. a EDI-OCT measurement of choroidal thickness (top-horizontal and bottom-vertical) in an undertreated patient with a disease evolution of 13 years, showing a much thinner choroid of $148.5 \pm 39.6 \mu \mathrm{m}$. b EDI-OCT measurement of choroidal thickness (top-horizontal and bottom-vertical) in an adequately treated patient with a disease evolution of 11 years,

noninvasive and easily performed. For the moment, the major drawback is the fact that EDI-OCT gives only information on the posterior pole in commercially available instruments, which might change, as technology will probably allow to examine systematically more peripheral fundus sectors.

\section{Swept source OCT (SS-OCT)}

The SS-OCT is a new generation Fourier-domain OCT that became available in clinical practice in 2012, with the introduction of the Topcon DRI OCT-1 Atlantis followed by the Topcon DRI OCT Triton (Tokyo, Japan) and by other newer devices. Its light source is a tuneable laser with a median wavelength of $1050 \mathrm{~nm}$. The $\mathrm{SS}-\mathrm{OCT}$ has increased scan speed (axial scan rate of $100,000 \mathrm{~Hz}$ ) and a deeper penetration than previous OCT modalities. The deeper penetration allows simultaneous clear and detailed visualization of the vitreous, the retina, and the choroid. This ultra-high speed technology allows wider fields of view up to $12 \times 12 \mathrm{~mm}$. These performances of SS-OCT showing a conserved thickness of $283 \pm 14.6 \mu \mathrm{m}$. c Fundus photography of an undertreated patient with a disease evolution of 13 years (same patient as in (a)) with numerous typical BRC lesions. d Fundus photography of an adequately treated patient with a disease evolution of 11 years (same patient as in (b)) with an absence of typical birdshot retinochoroiditis lesions.

make it a very useful tool for performing en face OCT and OCT-A [71-73]. In addition to that, SS-OCT can provide different windows for better vitreous visualization as well as segmentation and mapping of choroidal thickness.

The SS-OCT is characterized by improved penetration through opacities including vitritis, severe anterior segment inflammation, small pupil, and cataract, with better delineation of retinal layers in eyes with uveitis [22, 74]. Details are visualized on SS-OCT with higher resolution and greater span than conventional SD-OCT [74, 75] (Fig. 11).

Both EDI-OCT and SS-OCT seem to give comparable measurements of the sub-foveal choroidal thickness in eyes with VKH disease. However, SS-OCT is more likely to identify sclero-choroidal interface and less likely to produce unmeasurable images. It also enables imaging of the choroid and its vasculature in much greater detail [74]; thus it is especially useful in studies of choroidal vascularity index [76].

SS-OCT has shown to be very helpful in monitoring choroidal quantitative and qualitative changes during follow-up, assessing both response to treatment and disease activity or relapses [72, 74, 76-78]. 


\section{OCT angiography (OCT-A)}

While in the past all the OCT machines captured images like an in vivo biopsy by representing a sagittal slice of the retinal tissue, called B-scan analysis, and even tridimensional reconstructions, recently the technology turned towards the assessment of retinal vascular functionality,
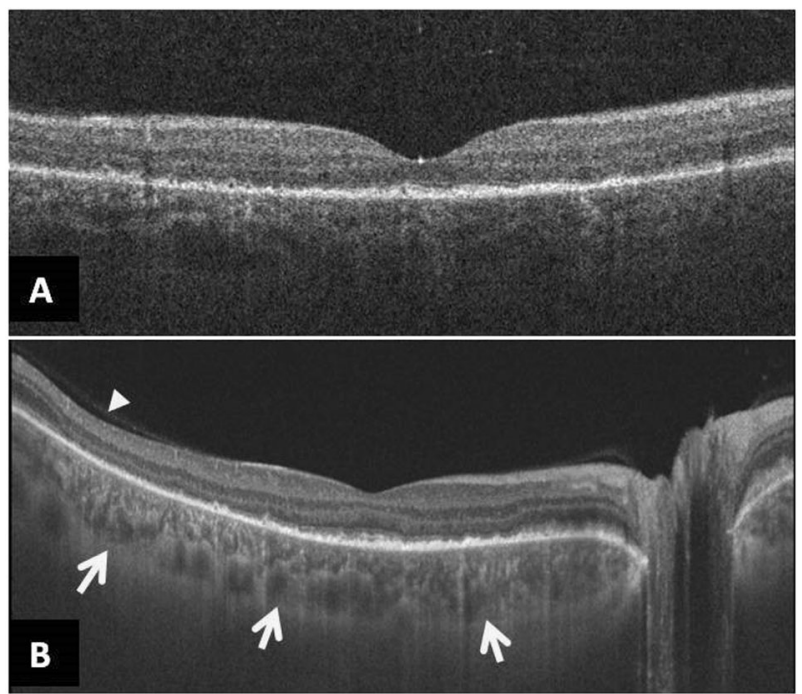

Fig. 11 Comparison of the B-scan images between SD-OCT and swept source OCT in a patient with chronic Vogt-KoyanagiHarada disease. Compared to SD-OCT (a), SS-OCT allows simultaneous visualization of the vitreous, the retina, and the choroid with a high resolution and a 12-mm view (b). The posterior hyaloid (arrowhead) and the sclero-choroidal interface (arrows).
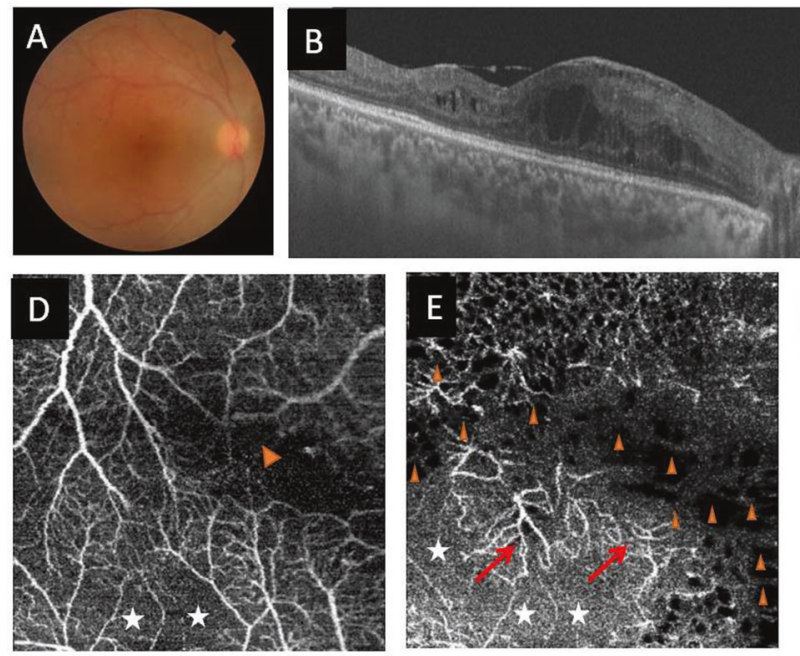

Fig. 12 Multimodal imaging of the right eye of a 32-year-old female patient with Behçet disease. a Colour fundus photography showing vascular sheathing; b swept source OCT B-scan showing macular oedema with cystoid spaces. c Composite fundus fluorescein angiogram of the same eye showing diffuse fern-like capillary leakage with associated peripheral nonperfused areas nasally and temporally. d $3 \times 3 \mathrm{~mm}$ swept source OCT angiography scan of the superficial through OCT-A [79]. This relatively novel noninvasive, dye-less imaging tool reconstructs high-resolution, depthresolved en face images of the retinal capillary networks by using a technology that compares the quantity of light returned from static and moving targets [79, 80]. Despite the undisputed role of FA in uveitis, which has been the gold standard for studying retinal hemorheology with the use of intravenous dye, one of the main limitations is the limited capability of FA to analyse the different retinal vascular layers. OCT-A may provide a precise analysis of superficial and deep retinal capillary networks by specific algorithms, allowing detection of differential involvement of the two layers that cannot be demonstrated by FA [81]. Although not yet validated nor standardized for routine use in uveitis, OCT-A can give valuable information.

\section{Retinal vascular changes}

OCT-A has become an important component of the multimodal imaging approach for the diagnosis and management in patients with uveitis, including retinal vasculitis [82, 83].

OCT-A allows qualitative and quantitative assessment of an array of retinal vascular changes in eyes with retinal vasculitis. These include areas of retinal capillary hypoperfusion, perifoveal capillary changes, including dilated, rarefied and shunting vessels, perifoveal capillary arcade disruption, enlargement of foveal avascular zone (FAZ), disorganization of capillary network, and decreased capillary vessel density (Fig. 12). Kim et al. [84] used a

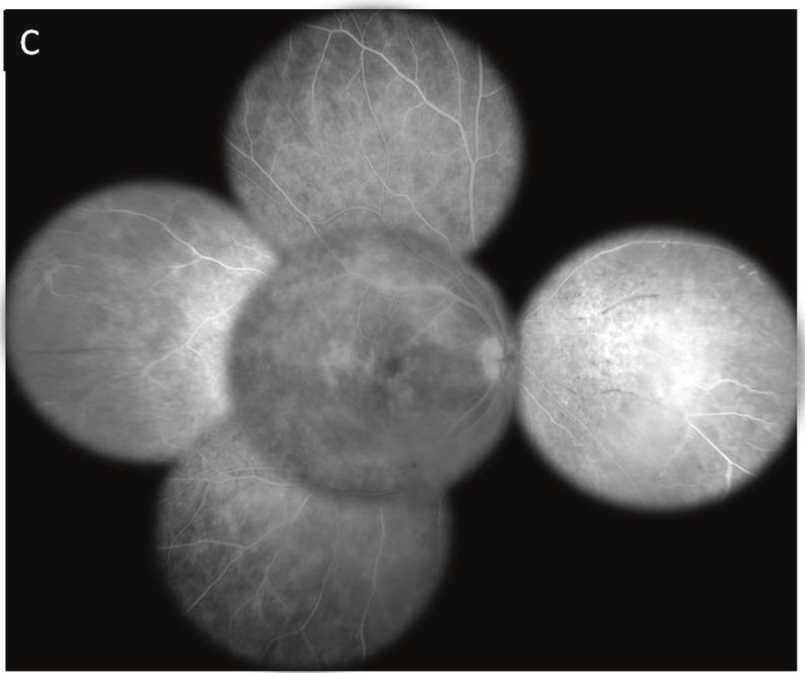

capillary plexus clearly visualizes well-delineated hypointense greyish areas of retinal capillary nonperfusion (white asterisk) and perifoveal capillary arcade disruption (arrowhead). e Swept source OCT angiogram shows in the deep capillary plexus, larger greyish areas of capillary nonperfusion (white asterisk), well-defined black roundish areas completely devoid of flow (arrowheads), and capillary telangiectasias (red arrows). 
prototype SD-OCT-A device (Cirrus, Carl Zeiss Meditec, Inc., Dublin, CA, USA) and reported that uveitis patients showed significantly lower branching complexity in the superficial retinal capillary plexus (SCP). Similarly, Bessete et al. [85] studied retinal vasculitis peculiarities by another OCT-A machine (Avanti RTVue-XR, Optovue Inc., Fremont, CA), reporting loss in the SCP, capillary dropout, abnormal FAZ morphology, as well as capillary remodelling. Interestingly, the deep retinal capillary plexus (DCP) presented minimal changes compared to the rest of the retinal vessels, albeit this might be due to confounding effects of projection artefacts. In fact, several studies have demonstrated a more severe involvement of the DCP in patients with retinal vasculitis, including Behçet disease and sarcoidosis $[86,87]$. However, the role of OCT-A in evaluating severity and in changing clinical practice patterns and decisions in the management of patients with retinal vasculitis is yet to be determined. Standard OCT-A has a limited field of view (FOV), and FA remains essential in showing vascular leakage, a key parameter of active inflammation, and in detecting peripheral ischaemia and neovascularization.

In eyes with uveitic macular oedema, OCT-A shows decreased retinal vascular density and increased FAZ in the DCP. [84, 88-90] Qualitative findings mainly include welldelineated cystoid spaces completely devoid of flow, greyish nonperfused or hypoperfused areas, rarefied capillaries, architectural disorganization, and dilated capillaries $[84,88]$. The resolution of uveitic CMO may be associated with a complete recovery of the normal pattern of retinal capillary plexuses or a persistence of capillary rarefaction or hypoperfusion on OCT-A. Thus, OCT-A findings provide a deeper insight into the pathophysiological processes of uveitic CMO [88].

\section{Choroidal inflammatory changes}

OCT-A may be helpful for diagnosis, and monitoring of several inflammatory conditions with choroidal involvement. It has also improved our understanding of the pathogenesis of such uveitic entities.

OCT-A can show choriocapillaris flow voids that may correspond to areas of true choriocapillaris ischaemia or to other alternative mechanisms. These include blocking or shadowing artefacts and space-occupying granulomas with or without associated choriocapillaris ischaemia [91]. In APMPPE, OCT-A typically shows flow reduction at the level of choriocapillaris or inner choroid. Low flow areas on OCT-A correspond to early hypofluorescence areas on ICGA. Decreased flow signal in the choriocapillaris on OCT-A B-scans have been demonstrated even in areas without any outer retinal inflammatory changes, excluding any shadowgraphic or projection artefacts (Fig. 13). These findings suggest a primary inner choroidal ischaemic involvement with secondary RPE and outer retinal changes. Follow-up OCT-A typically shows partial recovery of the choriocapillaris [92, 93]. Conversely, Pichi et al. [94] have reported a completely normal choriocapillaris flow in patients with MEWDS and proposed inflammation involving the outer photoreceptor level (photoreceptoritis), rather than the choricapillaris. These findings contradicting the admitted pathophysiology of MEWDS, can be explained by the fact that MEWDS is situated at the benign end of the choriocapillaris entities with closure of the endcapillaris where flow is so slow that OCT-A cannot detect whether there is flow or not and therefore appears as normal, while ICGA is clearly showing hypoperfusion [95]. Moreover, recent data have shown transient multifocal small areas of flow reduction at the level of the choriocapillaris in patients MEWDS, supporting the hypothesis of transient, primary or secondary choriocapillaris hypoperfusion [96]. Choriocapillaris flow voids may be secondary to choroidal granulomas (reduced flow due to full-thickness granuloma), or to focal choroidal arteriolitis in BRC, VKH disease, or sarcoidosis [87, 93, 97, 98].

\section{Inflammatory choroidal neovascularisation (CNV)}

OCT-A enables precise detection of inflammatory $\mathrm{CNV}$ lesions seen as a retino-choroidal microvascular network. Early detection of CNV may potentially lead to improved outcomes. In addition, OCT-A is helpful for monitoring response to treatment [99] (Fig. 14).

\section{New perspectives in imaging of posterior uveitis [ultra-high resolution, wide field, deeper penetration optical coherence tomography angiography (OCT-A)]}

Although this section is outside the scope of this review on validated and currently available imaging methods, it is justified to cite potential new developments.

One of the limitations encountered by the machines mentioned above was the exploration of retinal vascular periphery. Recently, a new OCT-A machine (Zeiss Plex Elite 9000, Carl Zeiss Meditec, Inc., Dublin, CA, USA) has introduced significant innovations for many clinical findings previously unexplored by an OCT-A device.

This new OCT-A is a transformational OCT imaging technique that uses swept source technology with faster scans acquired at $200 \mathrm{kHz}$ speed, exploring deeper components of the retinal-choroidal tissue, and with higher resolution that allows a fine discrimination of structural and microvascular components of retinal and choroidal anatomy. One of the main innovative aspects explored by this 

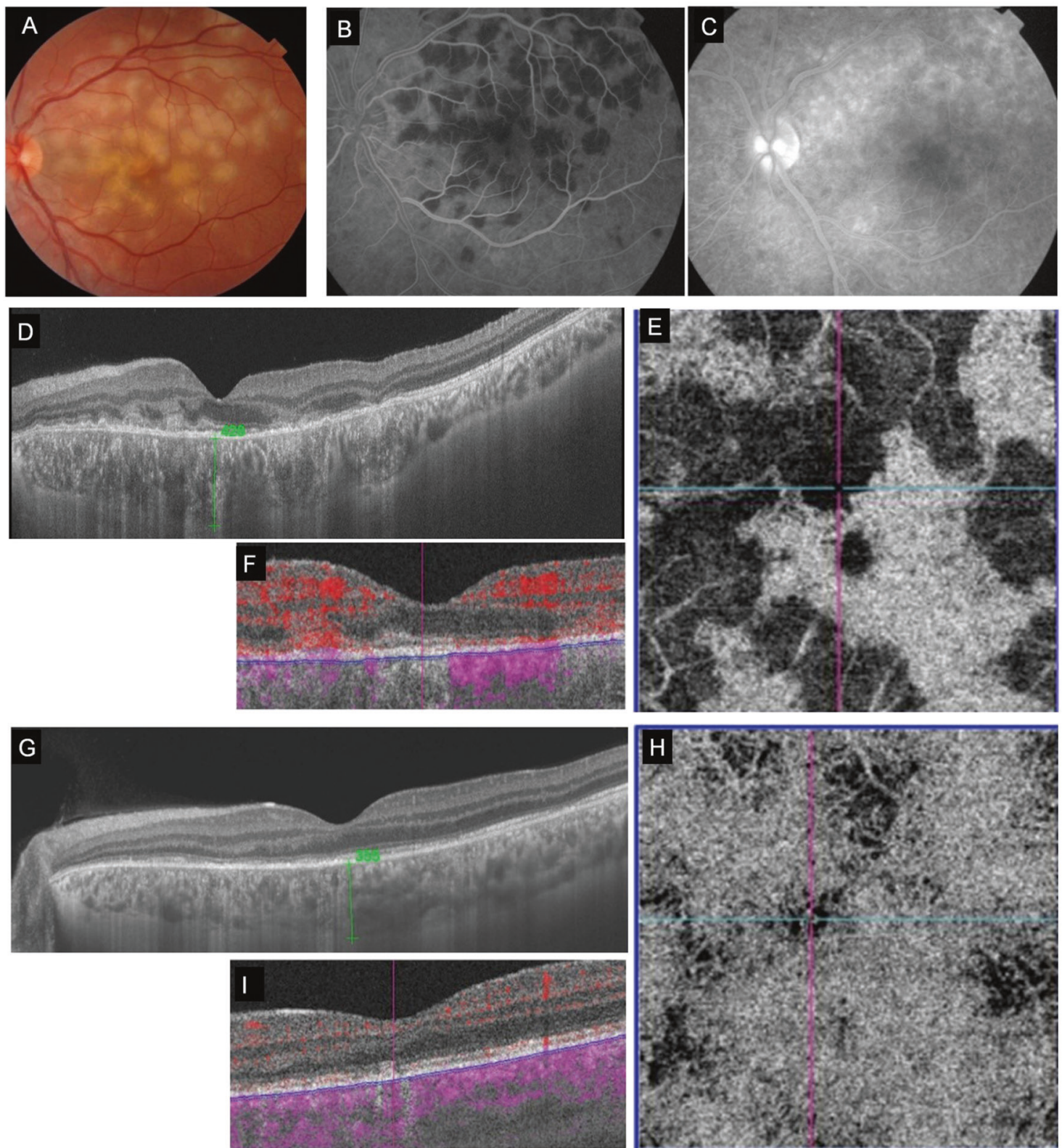

Fig. 13 Multimodal imaging of the left eye of a 23-year-old male patient with acute posterior multifocal placoid pigment epitheliopathy (APMPPE). a Fundus photograph showing white/grey subretinal placoid lesions in the posterior pole. b, c Fluorescein angiography shows numerous hypofluorescent lesions in early frames with staining in later frames. d Swept source OCT showing hyperreflective areas in the photoreceptor layer and alterations in the outer retinal layers (external limiting membrane, ellipsoid zone, and OS/

device is represented by the wide analysis which can provide two different montage OCT-A images: the first technique is a one central fixation for the acquisition of two ultra-high resolution OCT-A by $15 \times 9 \mathrm{~mm}$ scans, the second provides $70^{\circ} \mathrm{FOV}$ of the montage angiography by 5 OCT-A scans by $12 \times 12 \mathrm{~mm}$ in less than half the time (Fig. 15). At the moment, only one manuscript addresses this aspect: Tian et al. [100] used ultra-wide filed OCT-A
RPE (COST) junction). e En Face swept source OCT angiography revealing multifocal hypointense areas of reduced choriocapillaris flow with and without overlying inflammatory outer retinal changes on Bscan OCT (f). g Structural OCT, 1 month after initial presentation, showing improvement with persistent changes including photoreceptor atrophy, lack of reconstitution of the ellipsoid zone and OS/RPE lines. h, i Swept source OCT angiography, 1 month after presentation, showing significant improvement of choriocapillaris flow reduction.

for the evaluation of vascular changes in intermediate uveitis and retinal vasculitis. The authors concluded that changes in the choroidal structure, choriocapillaris and DCP were more relevant than SCP on wide-field OCTA in intermediate uveitis. However, wide-field OCTA was inefficient in determining disease activity that was well defined on FA instead. Under the light of such evidence, FA still remains the gold standard for studying vascular leakage, 

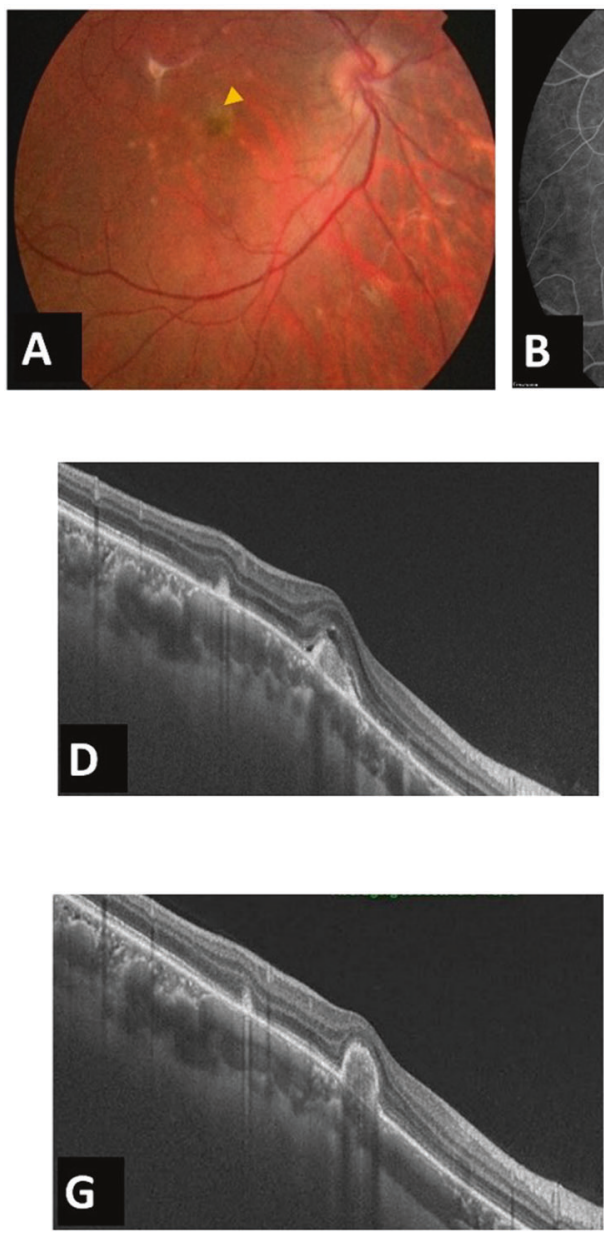

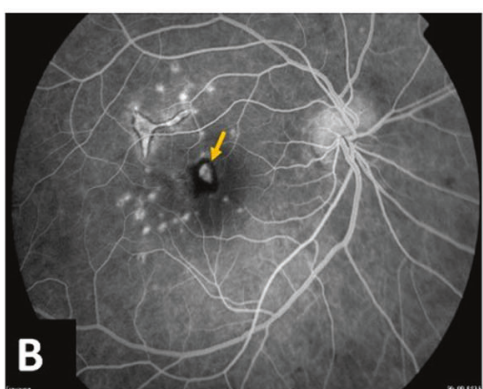

Angiography (Outer retina)

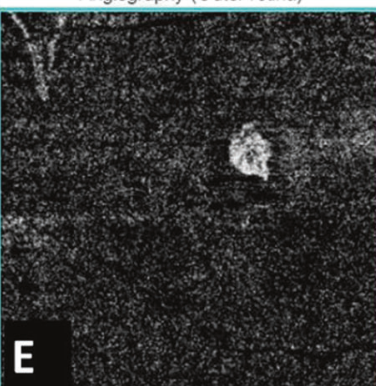

Angiography (Outer retina)

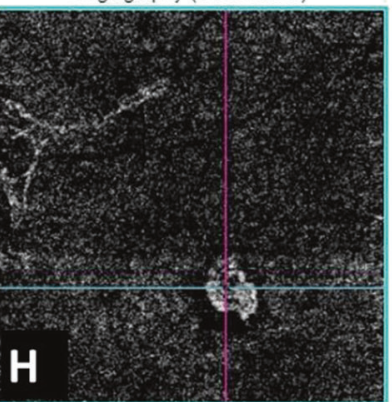

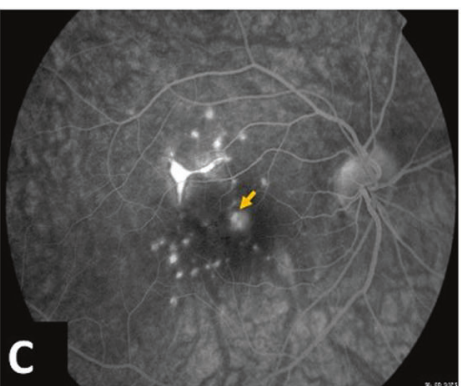

Angiography (Choriocapillaris)

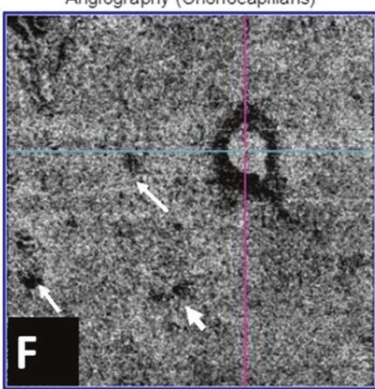

Angiography (Choriocapillaris)

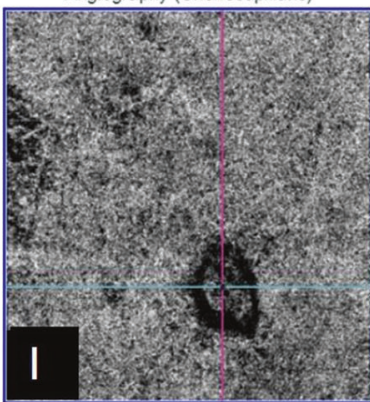

Fig. 14 Multimodal imaging of the right eye of a 27-year-old female patient with punctate inner choroidopathy. a Fundus photograph shows a greyish subretinal lesion (arrowhead). Early-phase (b) and late-phase (c) fluorescein angiograms show leakage due to choroidal neovascularization (yellow arrows). d Structural swept source OCT shows a fusiform subretinal hyperreflective lesion, associated with intraretinal fluid and serous retinal detachment. It also shows disruption of the ellipsoid and interdigitation zones associated with triangular-shape hyperreflective material over the RPE. e, f Swept source OCT angiography (OCTA) $3 \times 3 \mathrm{~mm}$ scan of the outer retina and choriocapillaris shows high-flow neovascular network corresponding to choroidal neovascular membrane (CNVM), area of surrounding hyporeflectivity around the CNVM associated with some areas of flow deficit corresponding to choroidal inflammatory lesions (white arrows). One month after a single intravitreal injection of bevacizumab, structural swept source OCT (g) shows resolution of serous retinal detachment and OCTA $(\mathbf{h}, \mathbf{i})$ reveals a decrease in the size of CNVM with a decrease in vessel calibre and branching.
Fig. 15 Widefield OCT-A imaging of a case of tuberculosis related occlusive frosted branch angiitis. Ultrawide-field colour fundus photography (a). The evident retinal ischaemia and the capillary drop down, as well as the vascular remodelling on OCT-A (b).
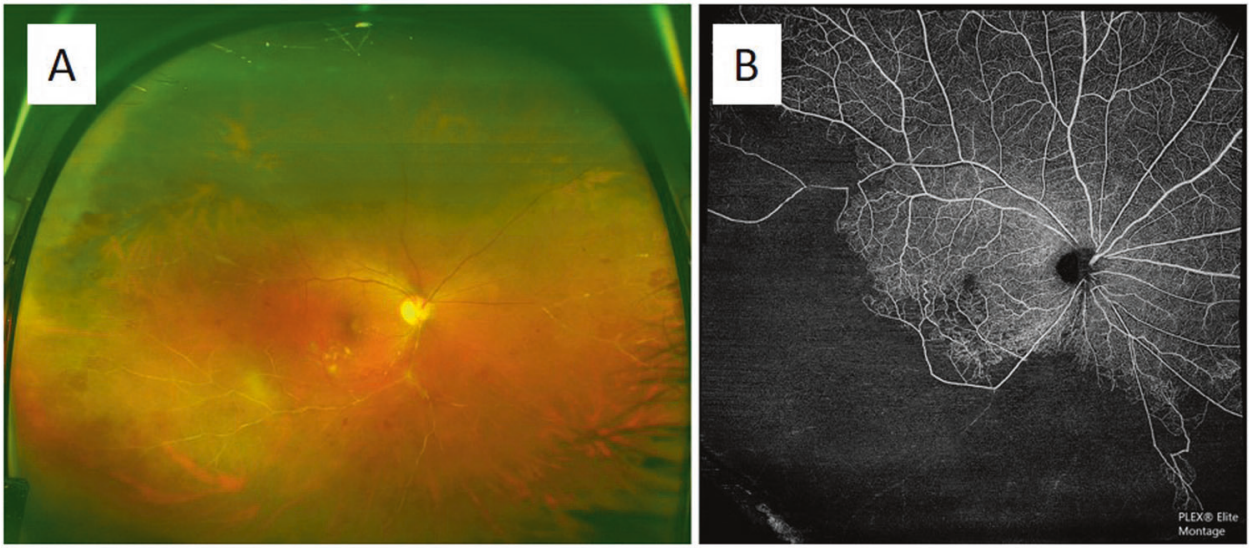
Fig. 16 Choroidal imaging in active choroidal stromal granulomata. There is a similar pattern of distribution of the choroidal lesions between ICGA (a) and OCT-A (b).
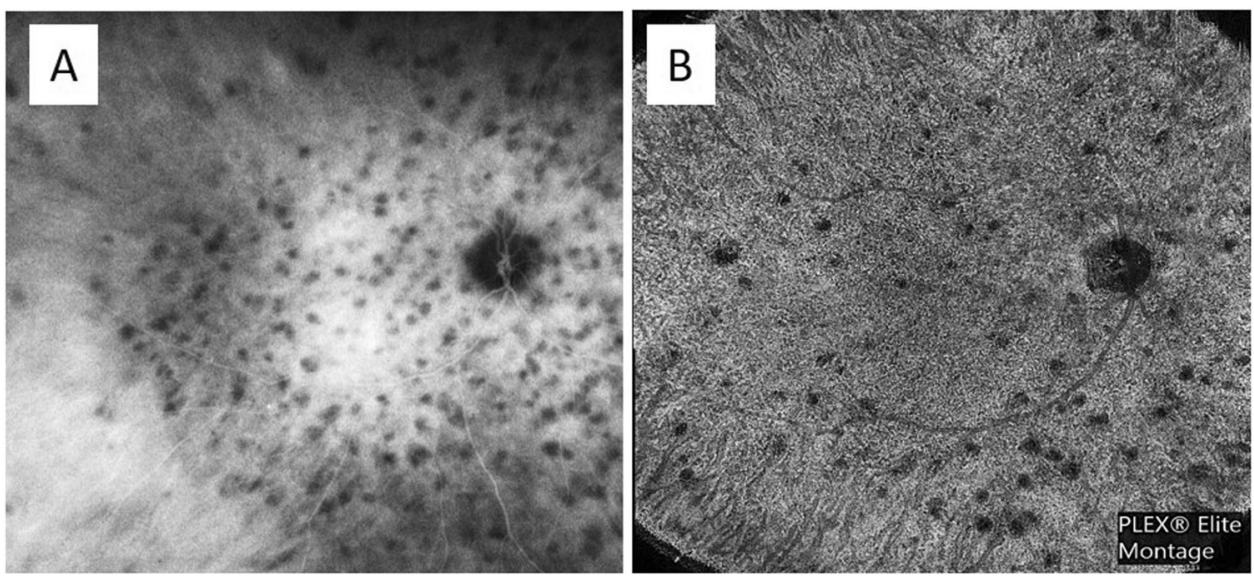

which represents one of the most important aspects for the identification and quantification of vasculitis activity. However, such new technology is just at the beginning of its potentiality exploration and more has to come for certain.

There is no doubt that choroidal analysis represents the most challenging aspect for OCT-A. Very little has been published till now on stromal choroidal exploration [91], due to the technical limitations of the first generation OCTA machines.

While it is undisputed that ICGA represents the gold standard for choroidal assessment, the new generation of SS-OCT-A has the capability to explore that component of the retinal-choroidal tissue. As per ICGA principles, choroidal space can present a decreased or even absent fluorescence (hypofluorescence) by the impaired filling of choroidal tissue due to space-occupying lesions, such as choroidal granulomata. A constant hypofluorescence for the whole angiogram duration is interpreted as a full-thickness invasion of choroidal stroma, while iso-fluorescence at the late phases is considered a sign of partial thickness choroidal space occupation.

In uveitis entities such as VKH disease, sympathetic ophthalmia, and HLA-A29 BRC that can present stromal choroidal lesions, the pathology might be explained by the following hypothesis: while at the beginning inflammatory cells progressively infiltrate connective tissue in between choroidal vascular structures leading to granulomatous choroidal arteriolitis, the natural progression of granulomata leads to the loss of choroidal connective tissue with shift and compression of the surrounding vascular tissue [101]. The technical characteristics of the new SS-OCT-A (Zeiss Plex Elite 9000, Carl Zeiss Meditec, Inc., Dublin, CA, USA) allow the visualization of all the different choroidal components from choriocapillaris up to supra-scleral space [102], providing images that may almost overlap the ones obtained by ICGA (Fig. 16). In addition, the width of the FOV has the same characteristics of the retinal OCT-A analysis described above, as well as it has the possibility to

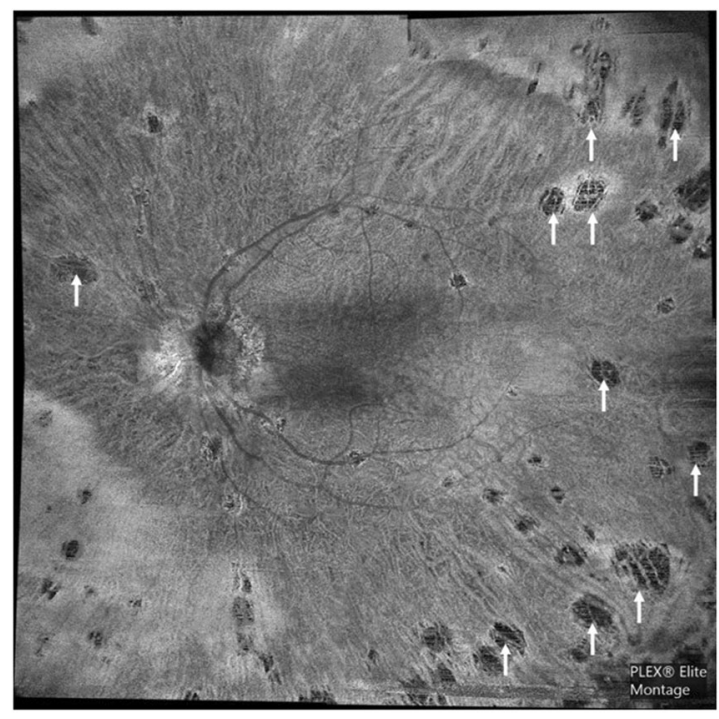

Fig. 17 OCT-A imaging of a case of inactive Vogt-KoyanagiHarada disease. The impaired stromal vascular tissue with voids into the spots where the active granulomata used to be in the active phase (white arrows).

crop the frontal images and analyse the stromal layers accurately at different depths. These images correlated with those provided by B-scan ultra-high-definition EDI-OCT can give a tridimentional multimodal OCT overview, giving an accurate analysis and a precise quantification of stromal choroidal tissue involvement. A further interesting finding seen in uveitic stromal choroidal lesions is the lack of support by intervascular stromal connective tissue in inactive choroiditis: it may be interpreted as impaired stromal vascular tissue (choroidal scars) with voids into the spots where the active granulomata used to be (Fig. 17).

In summary, new generation SS-OCT-A represents more than a promising tool for both retinal and choroidal assessment and its development will give an appropriate positioning to such new technology: further upcoming studies will provide sufficient evidence in order to be 
validated on a large scale. An additional hurdle would then be to have such techniques for general use.

\section{Conclusion}

Technologies for noninvasive imaging of the posterior segment have rapidly improved in the last two decades enabling fast, objective, and accurate assessment of posterior uveitis, which has led to improved diagnostic accuracy, better understanding of disease mechanisms, and precise monitoring of response to treatment. FAF and OCT imaging of the retina and choroid are essential components of multimodal imaging of inflammatory posterior segment pathologies. With improvements in OCT-A technology and standardization of OCT-A imaging, this technology may also become integrated in routine assessment of uveitis patients.

\section{Compliance with ethical standards}

Conflict of interest The authors declare that they have no conflict of interest.

Publisher's note Springer Nature remains neutral with regard to jurisdictional claims in published maps and institutional affiliations.

\section{References}

1. Nussenblatt RB, Palestine AG, Chan CC, Roberge F. Standardization of vitreal inflammatory activity in intermediate and posterior uveitis. Ophthalmology. 1985;92:467-71.

2. Jabs DA, Nussenblatt RB, Rosenbaum JT. Standardization of uveitis nomenclature (SUN) working group. Standardization of uveitis nomenclature for reporting clinical data. Results of the First International Workshop. Am J Ophthalmol. 2005;140: 509-16.

3. Mantovani A, Giani A, Herbort Jr CP, Staurenghi G. Interpretation of fundus autofluorescence changes in choriocapillaritis: a multi-modality imaging study. Graefes Arch Clin Exp Ophthalmol. 2016;254:1473-9.

4. Hedayatfar A, Khochtali S, Khairallah M, Takeuchi M, El Asrar AA, Herbort Jr CP. "Revised diagnostic criteria" for VogtKoyanagi-Harada disease fail to improve disease management. J Curr Ophthalmol. 2018;31:1-7.

5. Read RW, Holland GN, Rao NA, Tabbara KF, Ohno S, Arellanes-Garcia L, et al. Revised criteria for Vogt-KoyanagiHarada disease: report of an international committee on nomenclature. Am J Ophthalmol. 2001;131:647-52.

6. Herbort CP, Neri P, El Asrar AA, Gupta V, Kestelyn P, Khairallah $\mathrm{M}$, et al. Is ICGA still relevant in inflammatory eye disorders? Why this question has to be dealt with separately from other eye conditions. Retina. 2012;32:1701-3.

7. Fabro F, Herbort Jr CP. Need for quantitative measurement methods for posterior uveitis. Comparison of dual FA/ICGA angiography, EDI-OCT and SUN vitreous haze evaluation in stromal choroiditis. Klin Monbl Augenheilkd. 2018;235: 424-35.
8. Gillmann K, El Ameen A, Massy R, Fabro, Gasc A, Herbort Jr CP. Assessment of measurement methods of posterior inflammation in stromal choroiditis: the value of quantitative outcome measures versus the presently qualitatively based paradigm. Int Ophthalmol. 2019;39:1567-74.

9. Herbort Jr CP, Tugal-Tutkun I, Neri P, Pavésio C, Onal S, LeHoang $P$. Failure to integrate quantitative measurement methods of ocular inflammation hampers clinical practice and trials on new therapies for posterior uveitis. J Ocul Pharmacol Ther. 2017;33:263-77.

10. Tugal-Tutkun I, Cingü K, Kir N, Yeniad B, Urgancioglu M, Gül A. Use of laser flare-cell photometry to quantify intraocular inflammation in patients with Behçet uveitis. Graefes Arch Clin Exp Ophthalmol. 2008;246:1169-77.

11. Yalcindag FN, Bingol Kiziltunc P, Savku E. Evaluation of intraocular inflammation with laser flare photometry in Behçet uveitis. Ocul Immunol Inflamm. 2017;25:41-45.

12. Magone MT, Nussenblatt RB, Whitcup SM. Elevation of laser flare photometry in patients with cytomegalovirus retinitis and AIDS. Am J Ophthalmol. 1997;124:190-8.

13. Guex-Crosier Y, Pittet N, Herbort CP. Sensitivity of laser flare photometry to monitor inflammation in uveitis of the posterior segment. Ophthalmology. 1995;102:613-21.

14. Tugal-Tutkun I, Herbort CP. Laser flare photometry, a noninvasive objective and quantitative measure to measure intraocular inflammation. Int Ophthalmol. 2010;30:453-64.

15. Delori F, Keilhauer C, Sparrow JR, Staurenghi G. Origin of fundus autofluorescence. In: Holz FG, Schmitz-Valckenberg S, Spaide RF, Bird AC, editors. Atlas of fundus autofluorescence imaging. Heidelberg: Springer; 2007. p. 17-26.

16. Staurenghi G, Luiselli C, Levi G, Viola F, Delori FC. Retinal photopigment density measured using autofluorescence imaging. Investig Ophthalmol Vis Sci. 2004;45:e-abstract 2795.

17. Joseph A, Rahimy E, Freund KB, Sorenson JA, Sarraf D. Fundus autofluorescence and photoreceptor bleaching in multiple evanescent white dot syndrome. Ophthalmic Surg Lasers Imaging Retin. 2013;44:588-92.

18. Herbort Jr CP, Papasavvas I, Mantovani A. Choriocapillaris involvement in acute syphilitic posterior placoid chorioretinitis is responsible for functional impairment: a comprehensive clinicopathological approach. J Curr Ophthalmol. 2020; in press.

19. Boudreault KA, Schuerch K, Zhao J, Lee W, Cabral T, Yannuzzi LA, et al. Quantitative autofluorescence intensities in acute zonal occult outer retinopathy vs healthy eyes. JAMA Ophthalmol. 2017;135:1330-38.

20. Lee CS, Lee AY, Forooghian F, Bergstrom CS, Yan J, Yeh S. Fundus autofluorescence features in the inflammatory maculopathies. Clin Ophthalmol. 2014;8:2001-2012.

21. Huang D, Swanson EA, Lin CP, Schuman JS, Stinson WG, Chang W, et al. Optical coherence tomography. Science. 1991;254:1178-81.

22. Onal S, Tugal-Tutkun I, Neri P, Herbort CP. Optical coherence tomography imaging in uveitis. Int Ophthalmol. 2014;34: 401-35.

23. Markomichelakis NN, Halkiadakis I, Pantelia E, Peponis V, Patelis A, Theodossiadis $\mathrm{P}$, et al. Patterns of macular edema in patients with uveitis: qualitative and quantitative assessment using optical coherence tomography. Ophthalmology. 2004;111:946-53.

24. Hunter RS, Skondra D, Papaliodis G, Sobrin L. Role of OCT in the diagnosis and management of macular edema from uveitis. Semin Ophthalmol. 2012;27:236-41.

25. Grajewski RS, Boelke AC, Adler W, Meyer S, Caramoy A, Kirchhof B, et al. Spectral-domain optical coherence tomography findings of the macula in 500 consecutive patients with uveitis. Eye. 2016;30:1415-23. 
26. Dysli M, Rückert R, Munk MR. Differentiation of underlying pathologies of macular edema using spectral domain optical coherence tomography (SD-OCT). Ocul Immunol Inflamm. 2019;27:474-83.

27. Sugar EA, Jabs DA, Altaweel MM, Lightman S, Acharya N, Vitale AT, et al. Identifying a clinically meaningful threshold for change in uveitic macular edema evaluated by optical coherence tomography. Am J Ophthalmol. 2011;152:1044-.e5.

28. Grewal DS, O'Sullivan ML, Kron M, Jaffe GJ. Association of disorganization of retinal inner layers with visual acuity in eyes with uveitic cystoid macular edema. Am J Ophthalmol. 2017;177:116-25.

29. Lehpamer B, Moshier E, Pahk P, Goldberg N, Ackert J, Godbold $\mathrm{J}$, et al. Epiretinal membranes in uveitic macular edema: effect on vision and response to therapy. Am J Ophthalmol. 2014;157: 1048-55.

30. Duker JS, Kaiser PK, Binder S, de Smet MD, Gaudric A, Reichel E, et al. The International Vitreomacular Traction Study Group classification of vitreomacular adhesion, traction, and macular hole. Ophthalmology. 2013;120:2611-9.

31. Domalpally A, Altaweel MM, Kempen JH, Myers D, Davis JL, Foster CS, et al. Optical coherence tomography evaluation in the multicenter uveitis steroid treatment (MUST) trial. Ocul Immunol Inflamm. 2012;20:443-7.

32. Nicholson BP, Zhou M, Rostamizadeh M, Mehta P, Agron E, Wong W, et al. Epidemiology of epiretinal membrane in a large cohort of patients with uveitis. Ophthalmology. 2014;121: 2393-8.

33. Kahloun R, Gargouri S, Abroug N, Sellami D, Ben Yahia S, Feki $\mathrm{J}$, et al. Visual loss associated with rickettsial disease. Ocul Immunol Inflamm. 2014;22:373-8.

34. Tugal-Tutkun I, Ozdal PC, Oray M, Onal S. Review for diagnostics of the year: multimodal imaging in Behçet uveitis. Ocul Immunol Inflamm. 2017;25:7-19.

35. Oray M, Önal S, Koç Akbay A, Tuğal Tutkun I. Diverse clinical signs of ocular involvement in cat scratch disease. Turk J Ophthalmol. 2017;47:9-17.

36. Sivakumar RR, Prajna L, Arya LK, Muraly P, Shukla J, Saxena $\mathrm{D}$, et al. Molecular diagnosis and ocular imaging of West Nile virus retinitis and neuroretinitis. Ophthalmology. 2013;120: 820-1826.

37. Mahendradas P, Avadhani K, Shetty R. Chikungunya and the eye: a review. J Ophthalmic Inflamm Infect. 2013;3:35 https:// doi.org/10.1186/1869-5760-3-35.

38. Oray M, Onal S, Bayraktar S, Izgi B, Tugal-Tutkun I. Nonglaucomatous localized retinal nerve fiber layer defects in Behçet uveitis. Am J Ophthalmol. 2015;159:475-81.

39. Kido A, Uji A, Morooka S, Kuroda Y, Arichika S, Akagi T, et al. Outer plexiform layer elevations as a marker for prior ocular attacks in patients with Behcet's disease. Investig Ophthalmol Vis Sci. 2018;59:2828-32.

40. Invernizzi A, Agarwal AK, Ravera V, Mapelli C, Riva A, Staurenghi G, et al. Comparing optical coherence tomography findings in different aetiologies of infectious necrotising retinitis. Br J Ophthalmol. 2018;102:433-7.

41. Sikorski BL, Wojtkowski M, Kaluzny JJ, Szkulmowski M, Kowalczyk A. Correlation of spectral optical coherence tomography with fluorescein and indocyanine green angiography in multiple evanescent white dot syndrome. $\mathrm{Br} \mathrm{J}$ Ophthalmol. 2008;92:1552-7.

42. Li D, Kishi S. Restored photoreceptor outer segment damage in multiple evanescent white dot syndrome. Ophthalmology. 2009; 116:762-70.

43. Nazari Khanamiri H, Rao NA. Serpiginous choroiditis and infectious multifocal serpiginoid choroiditis. Surv Ophthalmol. 2013;58:203-32.
44. Zhang X, Zuo C, Li M, Chen H, Huang S, Wen F. Spectraldomain optical coherence tomographic findings at each stage of punctate inner choroidopathy. Ophthalmology. 2013;120: 2678-83.

45. Papadia M, Jeannin B, Herbort CP. OCT findings in birdshot chorioretinitis: a glimpse into retinal disease evolution. Ophthalmic Surg Lasers Imaging. 2012;43(Suppl):S25-31.

46. Yamaguchi Y, Otani T, Kishi S. Tomographic features of serous retinal detachment with multilobular dye pooling in acute VogtKoyanagi-Harada disease. Am J Ophthalmol. 2007;144:260-5.

47. Ishihara K, Hangai M, Kita M, Yoshimura N. Acute VogtKoyanagi-Harada disease in enhanced spectral-domain optical coherence tomography. Ophthalmology. 2009;116:1799-807.

48. Hosoda Y, Uji A, Hangai M, Morooka S, Nishijima K, Yoshimura N. Relationship between retinal lesions and inward choroidal bulging in Vogt-Koyanagi-Harada disease. Am J Ophthalmol. 2014;157:1056-63.

49. Spaide RF, Koizumi H, Pozonni MC. Enhanced depth imaging spectral-domain optical coherence tomography. Am J Ophthalmol. 2008;146:496-500.

50. Elahi S, Herbort Jr CP. Vogt-Koyanagi-Harada disease and birdshot retinochoroidopathy, similarities and differences: a glimpse into clinicopathology of stromal choroiditis, a perspective and a review. Klin Monbl Augenheilkd. 2019;236:492-510.

51. Behdad B, Rahmani S, Montahaei T, Soheilian R, Soheilian M. Enhanced depth imaging OCT (EDI-OCT) findings in acute phase of sympathetic ophthalmia. Int Ophthalmol. 2015;35: 433-9.

52. Invernizzi A, Agarwal A, Mapelli C, Nguyen QD, Staurenghi G, Viola F. Longitudinal follow-up of choroidal granulomas using enhanced depth imaging optical coherence tomography. Retina. 2017;37:144-53.

53. Nakai K, Gomi F, Ikuno Y, Yasuno Y, Nouchi T, Ohguro N, et al. Choroidal observation in Vogt-Koyanagi-Harada disease using high-penetration optical coherence tomography. Graefes Arch Clin Exp Ophthalmol. 2012;250:1089-95.

54. Nakayama M, Keino H, Okada AA, Watanabe T, Taki W, Inoue M, et al. Enhanced depth imaging optical coherence tomography of the choroid in Vogt-Koyanagi-Harada disease. Retina. 2012;32:2061-9.

55. Balci O, Gasc A, Jeannin B, Herbort Jr CP. Enhanced depth imaging is less suited than indocyanine green angiography for close monitoring of primary stromal choroiditis: a pilot report. Int Ophthalmol. 2017;37:737-48.

56. Balci O, Jeannin B, Herbort Jr CP. Contribution of dual fluorescein and indocyanine green angiography to the appraisal of posterior involvement in birdshot retinochoroiditis and VogtKoyanagi-Harada disease. Int Ophthalmol. 2018;38:527-39.

57. Elahi S, Gillmann K, Gasc A, Jeannin B, Herbort Jr CP. Sensitivity of indocyanine green angiography angiography compared to fluorescein and enhanced depth imaging optical coherence tomography during tapering and fine-tuning of therapy in primary stromal choroiditis: a case series. J Curr Ophthalmol. 2019;31:180-7.

58. Ishibazawa A, Kinouchi R, Minami Y, Katada A, Yoshida A. Recurrent Vogt-Koyanagi disease with sensorineural hearing loss and choroidal thickening. Int Ophthalmol. 2014;34:679-84.

59. Ikeda N, Ikeda T, Iwami H. Detection of asymptomatic choroiditis by fundus examination in Vogt-Koyanagi-Harada disease. Ocul Immunol Inflamm. 2017;25:830-3.

60. Tagawa Y, Namba K, Mizuuchi K, Takemoto Y, Iwata D, Uno $\mathrm{T}$, et al. Choroidal thickening prior to anterior recurrence in patients with Vogt-Koyanagi-Harada disease. Br J Ophthalmol. 2016;100:473-7.

61. Da Silva FT, Sakata VM, Nakashima A, Hirata CE, Olivalves E, Takahashi WY, et al. Enhanced depth imaging coherence 
tomography in long-standing Vogt-Koyanagi-Harada disease. $\mathrm{Br}$ J Ophthalmol. 2013;97:70-74.

62. Jap A, Chee SP. The role of enhanced depth imaging optical coherence tomography in chronic Vogt-Koyanagi-Harada disease. Br J Ophthalmol. 2017;101:186-9.

63. Murata T, Sako N, Takayama K, Harimoto K, Kanda K, Herbort $\mathrm{Jr} \mathrm{CP}$, et al. Identifying of underlying inflammation in VogtKoyanagi-Harada disease with sunset-glow fundus by multiple analysis. J Ophthalmol. 2019;2019:3853794. https://doi.org/10. 1155/2019/3853794.

64. Silpa-Archa S, Maleki A, Roohipoor R, Preble JM, Foster CS. Analysis of three-dimensional choroidal volume with enhanced depth imaging findings in patients with birdshot retinochoroidopathy. Retina. 2016;36:1758-66.

65. Boni C, Thorne JE, Spaide RF, Ostheimer TA, Sarraf D, Levinson RD, et al. Choroidal findings in eyes with birdshot chorioretinitis using enhanced-depth optical coherence tomography. Investig Ophthalmol Vis Sci. 2016;57:591-9.

66. Skvortsova N, Gasc A, Jeannin B, Herbort CP. Evolution of choroidal thickness over time and effect of early and sustained therapy in birdshot retinochoroiditis. Eye. 2017;31:1205-11.

67. El Ameen A, Herbort CP. Comparison of retinal and choroidal involvement in sarcoidosis-related chorioretinitis using fluorescein and indocyanine green angiography. $\mathrm{J}$ Ophthalmic Vis Res. 2018;13:426-32.

68. Ishihara M, Shibuya E, Tanaka S, Mizuki N. Diagnostic and therapeutic evaluation of multiple choroidal granulomas in a patient with confirmed sarcoidosis using enhanced depth imaging optical coherence tomography. Int Ophthalmol. 2018;38:2603-8.

69. Ishikawa S, Taguchi M, Muraoka T, Sakurai Y, Kanda T, Takeuchi M. Changes in subfoveal choroidal thickness associated with uveitis activity in patients with Behçet's disease. Br J Ophthalmol. 2014;98:1508-13.

70. Shirahama S, Kaburaki T, Nakahara H, Tanaka R, Komae K, Fujino Y, et al. Association between subfoveal choroidal thickness and leakage site on fluorescein angiography in Behçet's uveitis. Sci Rep. 2019;9:8612 https://doi.org/10.1038/s41598019-45149-4.

71. Lavinsky F, Lavinsky D. Novel perspectives on swept-source optical coherence tomography. Int J Retin Vitreous. 2016;2:25 https://doi.org/10.1186/s40942-016-0050-y.

72. Kishi S. Impact of swept source optical coherence tomography on ophthalmology. Taiwan J Ophthalmol. 2016;6:58-68. https:// doi.org/10.1016/j.tjo.2015.09.002.

73. Chen KC, Jung JJ, Engelbert M. Single acquisition of the vitreous, retina and choroid with swept-source optical coherence tomography in acute toxoplasmosis. Retin Cases Brief Rep. 2016;10:217-20.

74. Chee SP, Chan SN, Jap A. Comparison of enhanced depth imaging and swept source optical coherence tomography in assessment of choroidal thickness in Vogt-Koyanagi-Harada Disease. Ocul Immunol Inflamm. 2017;25:528-32.

75. Papavasileiou E, Miller JB, Sobrin L. Swept-source optical coherence tomography findings in convalescent phase of treated sarcoid choroidal granulomas. Retin Cases Brief Rep. 2016;10:32-6.

76. Agrawal R, Jain M, Khan R, Jaisankar D, Xin W, Ding J, et al. Choroidal structural changes in sympathetic ophthalmia on swept-source optical coherence tomography. Ocul Immunol Inflamm. 2019. https://doi.org/10.1080/09273948.2019.1685110.

77. Dastiridou AI, Bousquet E, Kuehlewein L, Tepelus T, Monnet D, Salah S, et al. Choroidal imaging with swept-source optical coherence tomography in patients with birdshot chorioretinopathy: choroidal reflectivity and thickness. Ophthalmology. 2017;124:1186-95.
78. Jaisankar D, Raman R, Sharma HR, Khandelwal N, Bhende M, Agrawal R, et al. Choroidal and retinal anatomical responses following systemic corticosteroid therapy in Vogt-KoyanagiHarada Disease using swept-source optical coherence tomography. Ocul Immunol Inflamm. 2019;27:235-243.

79. Spaide RF, Fujimoto JG, Waheed NK. Optical coherence tomography angiography. Retina. 2015;35:2161-2.

80. Agrawal R, Xin W, Keane PA, Chhableni J, Agarwal A. Optical coherence tomography angiography: a non-invasive tool to image end-arterial system. Expert Rev Med Dev. 2016;13: 519-21.

81. Abucham-Neto JZ, Torricelli AAM, Lui ACF, Guimarães SN, Nascimento H, Regatieri CV. Comparison between optical coherence tomography angiography and fluorescein angiography findings in retinal vasculitis. Int J Retin Vit. 2018;4:15 https:// doi.org/10.1186/s40942-018-0117-z.

82. Hassan M, Agarwal A, Afridi R, daSilva MJ, Karaca I, Sadiq MA, et al. The role of optical coherence tomography angiography in the management of uveitis. Int Ophthalmol Clin. 2016;56:1-24.

83. Agarwal A, Afridi R, Agrawal R, Do DV, Gupta V, Nguyen QD. Multimodal imaging in retinal vasculitis. Ocul Immunol Inflamm. 2017;25:424-33.

84. Kim AY, Rodger DC, Shahidzadeh A, Chu Z, Koulisis N, Burkemper B, et al. Quantifying retinal microvascular changes in uveitis using spectral domain optical coherence tomography angiography (SD-OCTA). Am J Ophthalmol. 2016;171:101-12.

85. Bessette AP, Levison AL, Baynes K, Lowder CY, Srivastava SK. Qualitative and quantitative analysis of optical coherence tomography angiography in patients with retinal vasculitis. Investig Ophthalmol Vis Sci. 2016;57:5504. (Abstract)

86. Khairallah M, Abroug N, Khochtali S, Mahmoud A, Jelliti B, Coscas G, et al. Optical coherence tomography angiography in patients with Behçet uveitis. Retina. 2017;37:1678-91.

87. Cerquaglia A, Iaccheri B, Fiore T, Fruttini D, Belli FB, Khairallah M, et al. New insights on ocular sarcoidosis: an optical coherence tomography angiography study. Ocul Immunol Inflamm. 2019;27:1057-66.

88. Khochtali S, Abroug N, Megzari K, Gargouri MA, Ksiaa I, Ben Amor $\mathrm{H}$, et al. Swept-source optical coherence tomography angiography findings in uveitic cystoid macular edema. Ocul Immunol Inflamm. 2019;27:1211-23.

89. Wintergerst MWM, Pfau M, Müller PL, Berger M, de Sisternes L, Holz FG, et al. Optical coherence tomography angiography in intermediate uveitis. Am J Ophthalmol. 2018;194:35-45.

90. Waizel M, Todorova MG, Terrada C, LeHoang P, Massamba N, Bodaghi B. Superficial and deep retinal foveal avascular zone OCTA findings of non-infectious anterior and posterior uveitis. Graefes Arch Clin Exp Ophthalmol. 2018;256:1977-84.

91. Pichi F, Sarraf D, Morara M, Mazumdar S, Neri P, Gupta V. Pearls and pitfalls of optical coherence tomography angiography in the multimodal evaluation of uveitis. J Ophthalmic Inflamm Infect. 2017;7:20 https://doi.org/10.1186/s12348-017-0138-z.

92. Klufas MA, Phasukkijwatana N, Iafe NA, Prasad PS, Agarwal A, Gupta V, et al. Optical coherence tomography angiography reveals choriocapillaris flow reduction in placoid chorioretinitis. Ophthalmol Retina. 2017;1:77-91.

93. Pichi F, Sarraf D, Arepalli S, Lowder CY, Cunningham ETJr, Neri $P$, et al. The application of optical coherence tomography angiography in uveitis and inflammatory eye diseases. Prog Retin Eye Res. 2017;59:178-201.

94. Pichi F, Srvivastava SK, Chexal S, Lembo A, Lima LH, Neri P, et al. En face optical coherence tomography and optical coherence tomography angiography of multiple evanescent white dot syndrome: new insights into pathogenesis. Retina. 2016;36 
(Suppl 1):S178-S188. https://doi.org/10.1097/IAE.00000000 00001255.

95. Lages V, Mantovani A, Papadia M, Herbort CP. MEWDS is a true choriocapillaritis and basic mechanisms do not differ from other choriocapillaris entities. J Curr Ophthalmol. 2018;30: 281-6.

96. Khochtali S, Dridi T, Abroug N, Ksiaa I, Lupidi M, Khairallah M. Swept-source optical coherence tomography angiography shows choriocapillaris flow reduction in multiple evanescent white dot syndrome. J Curr Ophthalmol. 2020; in press.

97. Pepple KL, Chu Z, Weinstein J, Munk MR, Van Gelder RN, Wang RK. Use of en face swept-source optical coherence tomography angiography in identifying choroidal flow voids in 3 patients with birdshot chorioretinopathy. JAMA Ophthalmol. 2018;136:1288-92.

98. Aggarwal K, Agarwal A, Mahajan S, Invernizzi A, Mandadi SKR, Singh R, et al. The role of optical coherence tomography angiography in the diagnosis and management of acute VogtKoyanagi-Harada disease. Ocul Immunol Inflamm. 2018;26: 142-153.

99. Astroz P, Miere A, Mrejen S, Sekfali R, Souied EH, Jung C, et al. Optical coherence tomography angiography to distinguish choroidal neovascularization from macular inflammatory lesions in multifocal choroiditis. Retina. 2018;38:299-309.

100. Tian M, Tappeiner C, Zinkernagel MS, Wolf S, Munk MR. Swept-source optical coherence tomography angiography reveals vascular changes in intermediate uveitis. Acta Ophthalmol. 2019;97:e785-e791. https://doi.org/10.1111/aos.14024.

101. Chen L, Xu G. Extensive choroidal infiltrates in choroidal biopsy proven ocular sarcoidosis. Retin Cases Brief Rep. 2013;7:69-70.

102. Pichi F, Invernizzi A, Tucker WR, Munk MR. Optical coherence tomography diagnostic signs in posterior uveitis. Prog Retin Eye Res. 2020;75:100797 https://doi.org/10.1016/j.preteyeres.2019. 100797. 